\title{
Null boundary controllability of a circular elastic arch
}

\author{
Arnaud $\mathrm{MÜNCH}$ *
}

October 26, 2009

\begin{abstract}
We consider a circular arch of thickness $\varepsilon$ and curvature $r^{-1}$ whose elastic deformations are described by a $2 \times 2$ system of linear partial differential equation. The system - of the type $\boldsymbol{y}^{\prime \prime}+\boldsymbol{A}^{\boldsymbol{\varepsilon}} \boldsymbol{y}=\mathbf{0}, \boldsymbol{y}=\left(y_{1}, y_{3}\right)$ - involves the tangential $y_{1}$ and normal $y_{3}$ component of the arch displacement. We analyze in this work the null controllability of these two components by acting on the boundary through a Dirichlet and a Neumann control simultaneously. Using the multiplier technic we show that, for any $\varepsilon>0$ fixed, the arch may be exactly controlled provided that the curvature be small enough. Then, we consider the numerical approximation of the controllability problem, using a $C^{0}-C^{1}$ finite element method and analyze some experiments with respect to the curvature and the thickness of the arch. We also highlight and discuss numerically the loss of uniform controllability as the thickness $\varepsilon$ goes to zero, due to apparition of an essential spectrum for the limit operator $\boldsymbol{A}^{\mathbf{0}}$.
\end{abstract}

Key Words. Arch equation, Null controllability, Dirichlet and Neumann control.

Mathematics Subject Classification. 35L05, 49J20, 65K10, 65M60, $93 \mathrm{~B} 05$.

\section{Introduction}

Let $\omega$ be a domain in $\mathbb{R}$ with boundary $\partial \omega, \varphi: \bar{\omega} \rightarrow \mathbb{R}^{2}$ a smooth function and $\varepsilon>0$. We consider an arch clamped on $\partial \omega$, made with homogeneous and isotropic, linear elastic material, with thickness $\varepsilon$ and whose middle surface is $\varphi(\bar{\omega})$. Submitted to applied forces with resultant $\boldsymbol{f}$ (expressed in a covariant basis attached to $\varphi(\bar{\omega}))$ and to initial condition $\left(\boldsymbol{y}^{\mathbf{0}}, \boldsymbol{y}^{\mathbf{1}}\right)$ at time $t=0$ the arch undergoes a displacement field $\boldsymbol{y}=\left(y_{1}, y_{3}\right)$ (expressed in the associated contravariant basis) which satisfies a system of linear partial differential equations:

$$
\left\{\begin{array}{lr}
\boldsymbol{y}^{\prime \prime}+\boldsymbol{A}^{\boldsymbol{\varepsilon}} \boldsymbol{y}=\boldsymbol{f} & \text { in } Q_{T}=\omega \times(0, T), \\
\boldsymbol{y}=\mathbf{0}, \partial_{\nu} y_{3}=0 & \text { on } \Sigma_{T}=\partial \omega \times(0, T), \\
\left(\boldsymbol{y}(\cdot, 0), \boldsymbol{y}^{\prime}(\cdot, 0)\right)=\left(\boldsymbol{y}^{\mathbf{0}}, \boldsymbol{y}^{\mathbf{1}}\right) & \text { in } \omega .
\end{array}\right.
$$

*Laboratoire de Mathématiques de Clermont-Ferrand, UMR CNRS 6620, Université Blaise-Pascal, Campus des Cézeaux, 63177 Aubière France, Email: arnaud.munch@math.univ-bpclermont.fr. Phone: $(+33)$ - 473407 054. Fax: (+33) - 473407 064. Partially supported by grants ANR-07-JCJC-0139-01 (Agence national de la recherche, France) and 08720/PI/08 from Fundacíon Séneca (Gobernio regional de Murcia, Spain). 
The symbol' denotes the partial differentiation with respect to time and $\boldsymbol{A}^{\varepsilon}$ a partial differential linear operator of the fourth order (depending on the map $\varphi$ and specified in the sequel). $\partial_{\nu} y_{3}$ designates the normal derivative of $y_{3}$.

We address in this work, on a theoretical and numerical viewpoint, the null controllability of the dynamical system (1): given any $T>0$ large enough and any initial condition $\left(\boldsymbol{y}^{\mathbf{0}}, \boldsymbol{y}^{\mathbf{1}}\right)$ in a suitable space, do there exists a function $\boldsymbol{v}=\left(v_{1}, v_{3}\right)$ acting on the system only through the boundary as follows:

$$
y_{1}=v_{1}, y_{3}=0, \partial_{\nu} y_{3}=v_{3} \quad \text { on } \Sigma_{T}
$$

such that

$$
\boldsymbol{y}(\cdot, T)=\boldsymbol{y}^{\prime}(\cdot, T)=\mathbf{0} \quad \text { in } \omega ?
$$

$y_{1}$ and $y_{3}$ denotes respectively the tangential and normal components of the displacement of the arch. $v_{1}$ acts on the tangential component and may be qualified as a Dirichlet control. $v_{3}$ acts on the derivative of the normal component and may be qualified as a Neumann control. Following linear arch and shell theory (we refer to [6], chapter 7), the operator $\boldsymbol{A}^{\varepsilon}$ is decomposed as follows:

$$
\boldsymbol{A}^{\varepsilon}=\boldsymbol{A}_{\boldsymbol{M}}+\frac{\varepsilon^{2}}{3} \boldsymbol{A}_{\boldsymbol{F}}
$$

where $\boldsymbol{A}_{\boldsymbol{M}}$ and $\boldsymbol{A}_{\boldsymbol{F}}$ designate the membrane and flexural operator respectively.

In spite of several works in the eighties about the controllability of beam and plate (see [17] and the references therein), much less is known in the case of the shell and rod. In this case, due to the coupling provided by the curvature, the study is more involved and most of the existing works deal with specific examples of the map $\varphi$. Thus, in $[9,11]$, Geymonat, Loreti and Valente show the exact controllability of an axi-symmetrical spherical cap for a small enough curvature. In [10], the same authors consider also the case of an hemispherical shell and get controllability using harmonic analysis. For these two cases the analysis highlights the loss of exact controllability in the transition shell-membrane, i.e. when the thickness $\varepsilon$ tends to zero. From the analytical expression of the eigenvalues of $\boldsymbol{A}^{\varepsilon}$ for the hemi-spherical, it appears that the spectrum of $\boldsymbol{A}^{\varepsilon}$ does not fulfill a uniform gap condition with respect to $\varepsilon$ and exhibit a minimal time of controllability $T_{\min }$ of order $O\left(\varepsilon^{-1}\right)$. Moreover, the operator $\boldsymbol{A}_{\boldsymbol{M}}$ has a non empty essential spectrum $\sigma_{\text {ess }}\left(\boldsymbol{A}_{\boldsymbol{M}}\right)=\{a\}, a>0$. This non-controllability result is extended in [12] to more general operators. According to this property, relaxed exact spectral [24] or partial [16] controllability of membrane shells have been studied. For $\varepsilon>0$, we also mention [20] where a controllability result is obtained for a shallow Koiter's shell using the Hilbert Uniqueness Method [18] and more recently [15] in the context of so-called intrinsic shell model. Furthermore, on the related thematic of stabilization, let us mention [4, 14] where dissipative terms are added on the shell model in order to obtain a specific decay of the corresponding energy. The boundary stabilization of a nonlinear arch is studied in [22].

From a numerical viewpoint, few works are available for mainly two reasons. First, 
independently of the controllability problem, the numerical approximation of arch and shell (by finite element method) remains difficult because of the locking phenomenon that may appear in the flexural case (we refer to [5], chapter 3). Secondly, since the pioneering work of Glowinski et al [13] (see [25] for a recent review), the exact controllability for hyperbolic systems is known to be sensitive to numerical approximation.

In this numerically oriented work, we address the exact controllability issue in the simple but rich case of a circular arch with the aim of producing some numerical experiments and discuss the sensitivity of the control with respect to the parameters, in particular the curvature and the thickness. In Section 2, we define precisely the operator $\boldsymbol{A}^{\boldsymbol{\varepsilon}}$ and state some existence results for the corresponding static formulation (Lemma 2.2). Similar qualitative results are given for the homogeneous dynamic problem (i.e. without control) in Section 3.1 (Lemma 3.1). In Section 3.2, we use the multiplier technic to obtain respectively the so-called hidden -regularity inequality (Theorem 3.1) and the observability inequality (Theorem 3.2), both involving the energy of the homogeneous solution. Then, Section 4, using the Hilbert Uniqueness Method introduced by J.-L. Lions in [18], these estimates permit to construct explicitly a control, assuming the curvature of the elastic arch small enough (see Theorem 4.1). In Section 5, we then address the numerical approximation of this control and study the influence of the curvature and thickness. The assumption on the curvature appears weak in practice (Section 5.2). Moreover, we numerically check, Sectionsec-influence-epsilon, the loss of uniform controllability as the thickness $\varepsilon$ goes to zero. As explained, this phenomenon is due to the fact that the limit operator $\boldsymbol{A}_{\boldsymbol{M}}$ posseses an essential spectrum: precisely, $0 \in \sigma_{\text {ess }}\left(\boldsymbol{A}_{\boldsymbol{M}}\right)$. Then, we observe that the uniform controllability is not recovered for initial datum $\left(\boldsymbol{y}^{\mathbf{0}}, \boldsymbol{y}^{\mathbf{1}}\right) \in\left(\operatorname{Ker} \boldsymbol{A}_{\boldsymbol{M}}\right)^{\perp}$. This is related to the non convergence of $\sigma\left(\boldsymbol{A}^{\boldsymbol{\varepsilon}}\right)$ toward $\sigma\left(\boldsymbol{A}_{\boldsymbol{M}}\right)$, enhanced by the degeneracy of the boundary conditions on $y_{3}$. Section 6 concludes this work with some perspectives and Appendix 7 analyzes the spectrum of $\boldsymbol{A}^{\varepsilon}$ and exhibits its densification over $\mathbb{R}^{+}$as $\varepsilon \rightarrow 0$, responsible of the lack of uniform spectral gap property.

\section{Static problem}

We explicit in this section the operator $\boldsymbol{A}^{\varepsilon}$ in the case of a circular arch and then state some important existence and regularity results in the static case.

\subsection{The case of a cylindrical arch}

Without loss of generality, let us assume that the mid-surface of the arch is $\omega=(0,1)$ and note by $\xi \in \omega$ the curvilinear abscissa. For a cylindrical arch, the map $\varphi: \bar{\omega} \rightarrow \mathbb{R}^{2}$ may takes the following expression

$$
\boldsymbol{\varphi}(\xi)=\left(r \sin \left(r^{-1} \xi\right), r \cos \left(r^{-1} \xi\right)\right)=\boldsymbol{x}=\left(x_{1}, x_{3}\right)
$$

where $r>0$ denotes the constant radius of curvature of the arch. The curvature is then $r^{-1}$. Remark that the length of the arch is equal to 1 for all $r>0$. The tangent vector $\boldsymbol{\tau}=\partial_{\xi} \boldsymbol{\varphi}$ 
and the normal vector $\boldsymbol{\nu}$ take the expression

$$
\left.\boldsymbol{\tau}=\left(\cos \left(r^{-1} \xi\right),-\sin \left(r^{-1} \xi\right)\right), \quad \boldsymbol{\nu}=\left(\sin \left(r^{-1} \xi\right)\right), \cos \left(r^{-1} \xi\right)\right)
$$

and any displacement vector $\boldsymbol{y}(\boldsymbol{x})$ of component $\left(\hat{y}_{1}, \hat{y}_{3}\right)$ in the orthonormal basis $\left(\boldsymbol{e}_{\mathbf{1}}, \boldsymbol{e}_{\mathbf{3}}\right)$ of $\mathbb{R}^{2}$ so that $\boldsymbol{y}(\boldsymbol{x})=\hat{y}_{1}(\boldsymbol{x}) \boldsymbol{e}_{\mathbf{1}}+\hat{y}_{3}(\boldsymbol{x}) \boldsymbol{e}_{\mathbf{3}}$ may then be expressed as follows

$$
\boldsymbol{y}(\boldsymbol{x})=y_{1}(\xi) \boldsymbol{\tau}(\xi)+y_{3}(\xi) \boldsymbol{\nu}(\xi), \quad \xi \in \omega
$$

in the contravariant basis $(\boldsymbol{\tau}, \boldsymbol{\nu})$ (which coincides here with the covariant basis). The operator $\boldsymbol{A}^{\varepsilon}$ is then defined by $\boldsymbol{A}^{\varepsilon}=\boldsymbol{A}_{\boldsymbol{M}}+\varepsilon^{2} / 3 \boldsymbol{A}_{\boldsymbol{F}}$ where

$$
\boldsymbol{A}_{\boldsymbol{M}} \boldsymbol{y}=a\left(\begin{array}{c}
-\left(y_{1,1}+r^{-1} y_{3}\right)_{, 1} \\
r^{-1}\left(y_{1,1}+r^{-1} y_{3}\right)
\end{array}\right)
$$

and

$$
\boldsymbol{A}_{\boldsymbol{F}} \boldsymbol{y}=a\left(\begin{array}{l}
2 r^{-1}\left(y_{3,11}-2 r^{-1} y_{1,1}-r^{-2} y_{3}\right)_{, 1} \\
y_{3,1111}-2 r^{-1} y_{1,111}-2 r^{-2} y_{3,11}+2 r^{-3} y_{1,1}+r^{-4} y_{3}
\end{array}\right) .
$$

$a$ designates the longitudinal elasticity coefficient of the arch. For simplicity, we take $a=1$ in the sequel. Moreover, $y_{i, 1}$ denotes the partial derivative of $y_{i}$ with respect to the curvilinear abcissa $\xi: y_{i, 1}(\xi, t)=\partial y_{i}(\xi, t) / \partial \xi, i \in\{1,3\}$.

Remark 1 When the curvature is assumed to be small $\left(r^{-1}<1\right)$, one may simplify the operator $\boldsymbol{A}_{\boldsymbol{F}}$ as follows:

$$
\boldsymbol{A}_{\boldsymbol{F}}^{\star} \boldsymbol{y}=a\left(\begin{array}{c}
r^{-1}\left(y_{3,1}-r^{-1} y_{1}\right), 11 \\
\left(y_{3,1}-r^{-1} y_{1}\right)_{, 111}
\end{array}\right)
$$

When the curvature is assumed to be very small $\left(r^{-1}<<1\right)$, one may consider simply

$$
\boldsymbol{A}_{\boldsymbol{F}}^{\star \star} \boldsymbol{y}=a\left(\begin{array}{l}
0 \\
y_{3,1111}
\end{array}\right)
$$

The operator $\boldsymbol{A}_{\boldsymbol{M}}$ remains unchanged in both cases. We refer for instance to [8], chapter 4.

Remark 2 The curvature $r^{-1}$ is the coupling parameter between the two components $y_{1}$ and $y_{3}$, so that when $r^{-1}=0$, the components are respectively solution of the wave and beam equation

$$
y_{1}^{\prime \prime}-y_{1,11}=0, \quad y_{3}^{\prime \prime}+\frac{\varepsilon^{2}}{3} y_{3,1111}=0 \quad \text { in } Q_{T} .
$$

The first equation is null controllable for any $T>1$ and $\left(y_{1}^{0}, y_{1}^{1}\right) \in L^{2}(\omega) \times H^{-1}(\omega)$ through $y_{1}=v_{1} \in L^{2}\left(\Sigma_{T}\right)$ (see [18], chapter 3). For any $\varepsilon>0$, the second equation is null controllable for any $T>0$ and $\left(y_{3}^{0}, y_{3}^{1}\right) \in L^{2}(\omega) \times H^{-2}(\omega)$ through $\partial_{\nu} y_{3}=v_{3} \in L^{2}\left(\Sigma_{T}\right)$, 
taking $y_{3}=0$ on $\Sigma_{T}$ (see [18], chapter 5).

Before to proceed to the analysis of the null controllability of system 1, let us insist on the following point. In the two-dimensional shell theory as presented in detail in $[6,7]$, the mid-surface $\omega$ belongs to $\mathbb{R}^{2}$ and systems involves three components of the displacement $\left(u_{1}, u_{2}, u_{3}\right)$. The operator $\boldsymbol{A}_{\boldsymbol{M}}$ and $\boldsymbol{A}_{\boldsymbol{F}}$ given in that section are derived from the $2-d$ case by simply eliminating the variable $u_{2}$. Moreover, the operator $\boldsymbol{A}^{\boldsymbol{\epsilon}}=\boldsymbol{A}_{\boldsymbol{M}}+\epsilon^{2} / 3 \boldsymbol{A}_{\boldsymbol{F}}$ appears in the well-admitted Koiter shell model (we refer to $[6,7]$ for the analysis of the Koiter shell model).

\subsection{Some results for the static problem}

In order to fix the notations, we summarize here some important results about the static problem : find $\boldsymbol{u}=\left(u_{1}, u_{3}\right)$ such that

$$
\left\{\begin{array}{l}
\boldsymbol{A}^{\varepsilon} \boldsymbol{u}=\boldsymbol{f} \\
\boldsymbol{u}=0, \partial_{\nu} u_{3}=0 \quad \text { in } \quad \omega,
\end{array}\right.
$$

Definition 2.1 For any $\boldsymbol{v}=\left(v_{1}, v_{3}\right)$ and $\boldsymbol{u}=\left(u_{1}, u_{3}\right)$, we introduce the following notation:

$$
\left\{\begin{array}{l}
\gamma(\boldsymbol{v})=v_{1,1}+r^{-1} v_{3}, \\
\rho(\boldsymbol{v})=v_{3,11}-r^{-1} v_{1,1}-r^{-1} \gamma(\boldsymbol{v})=v_{3,11}-2 r^{-1} v_{1,1}-r^{-2} v_{3} \\
b_{M}(\boldsymbol{u}, \boldsymbol{v})=\gamma(\boldsymbol{u}) \gamma(\boldsymbol{v}), \quad b_{F}(\boldsymbol{u}, \boldsymbol{v})=\rho(\boldsymbol{u}) \rho(\boldsymbol{v}), \quad b^{\varepsilon}(\boldsymbol{u}, \boldsymbol{v})=b_{M}(\boldsymbol{u}, \boldsymbol{v})+\frac{\varepsilon^{2}}{3} b_{F}(\boldsymbol{u}, \boldsymbol{v})
\end{array}\right.
$$

$\gamma$ is the linearized change of metric function associated with the map $\varphi . \rho$ is the linearized change of curvature function.

From these definitions, we may rewrite the operator $\boldsymbol{A}_{\boldsymbol{M}}$ and $\boldsymbol{A}_{\boldsymbol{F}}$ defined in (3) and (4) respectively in term of $\gamma$ and $\rho$ :

$$
\boldsymbol{A}_{\boldsymbol{M}} y=\left(\begin{array}{r}
-\gamma(\boldsymbol{y})_{, 1} \\
r^{-1} \gamma(\boldsymbol{y})
\end{array}\right), \quad \boldsymbol{A}_{\boldsymbol{F}} y=\left(\begin{array}{r}
2 r^{-1} \rho(\boldsymbol{y})_{, 1} \\
\rho(\boldsymbol{y})_{, 11}-r^{-2} \rho(\boldsymbol{y})
\end{array}\right)
$$

Then, computations based on integrations by parts, lead to the following relations (we refer to [20] for similar developments for shell operator).

Lemma 2.1 For all $\boldsymbol{u} \in H^{3}(\omega) \times H^{4}(\omega)$, we have,

- For all $\boldsymbol{v} \in H^{1}(\omega) \times H^{2}(\omega)$

$$
\begin{aligned}
& \int_{\omega} \boldsymbol{A}_{M} \boldsymbol{u} \cdot \boldsymbol{v} d \xi=\int_{\omega} b_{M}(\boldsymbol{u}, \boldsymbol{v}) d \xi-\left[\gamma(\boldsymbol{u}) v_{1}\right]_{0}^{1} \\
& \int_{\omega} \boldsymbol{A}_{\boldsymbol{F}} \boldsymbol{u} \cdot \boldsymbol{v} d \xi=\int_{\omega} b_{F}(\boldsymbol{u}, \boldsymbol{v}) d \xi+\left[\rho(\boldsymbol{u})_{, 1} v_{3}\right]_{0}^{1}-\left[\rho(\boldsymbol{u}) v_{3,1}\right]_{0}^{1}+2 r^{-1}\left[\rho(\boldsymbol{u}) v_{1}\right]_{0}^{1}
\end{aligned}
$$


- For all $\boldsymbol{v} \in H_{0}^{1}(\omega) \times H_{0}^{2}(\omega)$

$$
\int_{\omega} \boldsymbol{A}_{\boldsymbol{M}} \boldsymbol{u} \cdot \boldsymbol{v} d \xi=\int_{\omega} b_{M}(\boldsymbol{u}, \boldsymbol{v}) d \xi, \quad \int_{\omega} \boldsymbol{A}_{\boldsymbol{F}} \boldsymbol{u} \cdot \boldsymbol{v} d \xi=\int_{\omega} b_{F}(\boldsymbol{u}, \boldsymbol{v}) d \xi
$$

therefore

$$
\int_{\omega} \boldsymbol{A}^{\varepsilon} \boldsymbol{u} \cdot \boldsymbol{v} d \xi=\int_{\omega} b^{\varepsilon}(\boldsymbol{u}, \boldsymbol{v}) d \xi
$$

We now introduce the space $\boldsymbol{V}=H_{0}^{1}(\omega) \times H_{0}^{2}(\omega)$. As a consequence of the Poincaré's inequality on $\varphi(\bar{\omega})$ (see [6], chapter 4 for the counterpart Korn's inequality for shell), the energy norm

$$
\|\boldsymbol{v}\|_{\boldsymbol{V}}=\left(\int_{\omega} b^{\varepsilon}(\boldsymbol{v}, \boldsymbol{v}) d \xi\right)^{1 / 2}
$$

is a norm over $\boldsymbol{V}$. Therefore, the symmetric bilinear form $(\boldsymbol{u}, \boldsymbol{v}) \in \boldsymbol{V} \rightarrow \int_{\omega} b^{\varepsilon}(\boldsymbol{u}, \boldsymbol{v}) d \xi$ is continuous and $\boldsymbol{V}$-coercive so that Lax-Milgram theorem leads to the following result (see for instance [6]).

Lemma 2.2 (i) If $\boldsymbol{f} \in\left(L^{2}(\omega)\right)^{2}$, there exists a unique weak solution $\boldsymbol{u} \in \boldsymbol{V}$ to the variational problem

$$
\int_{\omega} b^{\varepsilon}(\boldsymbol{u}, \boldsymbol{v}) d \xi=\int_{\omega} \boldsymbol{f} \cdot \boldsymbol{v} d \xi, \quad \forall \boldsymbol{v} \in \boldsymbol{V}
$$

(ii) Let $\boldsymbol{E}=\left(H^{3}(\omega) \cap H_{0}^{1}(\omega)\right) \times\left(H^{4}(\omega) \cap H_{0}^{2}(\omega)\right)$. If $\boldsymbol{f} \in H^{1}(\omega) \times L^{2}(\omega)$, the unique solution $\boldsymbol{u}$ of (6) belongs to $\boldsymbol{E}$ and satisfies the estimates

$$
\exists C>0:\|\boldsymbol{u}\|_{\boldsymbol{E}} \leq C\left(\|\boldsymbol{f}\|_{H^{1}(\omega) \times L^{2}(\omega)}+\|\boldsymbol{u}\|_{L^{2}(\omega) \times L^{2}(\omega)}\right) .
$$

\section{Homogeneous evolution problem}

The homogeneous problem plays an important role in the Hilbert Uniqueness Method. We first recall some basic results and then derive several technical equalities related to the multiplier method.

We introduce the space $\boldsymbol{H}=L^{2}(\omega) \times L^{2}(\omega)$ endowed with the usual norm and identified to its dual space. The Hilbert space $\boldsymbol{V}=H_{0}^{1}(\omega) \times H_{0}^{2}(\omega)$ is dense in $\boldsymbol{H}$ with compact imbedding. Let $\boldsymbol{V}^{\prime}=H^{-1}(\omega) \times H^{-2}(\omega)$ denote the dual space of $\boldsymbol{V}$, so that $\boldsymbol{V} \subset \boldsymbol{H} \subset \boldsymbol{V}^{\prime}$.

\subsection{Existence - Uniqueness}

Let $T>0$ be given. We consider in this section the uncontrolled system

$$
\left\{\begin{array}{lr}
\boldsymbol{u}^{\prime \prime}+\boldsymbol{A}^{\varepsilon} \boldsymbol{u}=\boldsymbol{f} & \text { in } Q_{T}=\omega \times(0, T), \\
\boldsymbol{u}=\mathbf{0}, \partial_{\nu} u_{3}=0 & \text { on } \Sigma_{T}=\partial \omega \times(0, T), \\
\left(\boldsymbol{u}(\cdot, 0), \boldsymbol{u}^{\prime}(\cdot, 0)\right)=\left(\boldsymbol{u}^{\mathbf{0}}, \boldsymbol{u}^{\mathbf{1}}\right) & \text { in } \omega .
\end{array}\right.
$$

The existence, uniqueness and regularity of the solution of (8) is given by the next lemma, 
direct consequence of Lions's "abstract" theorem (we refer to [19]) and of the regularity given by Lemma 2.2 .

Lemma 3.1 (i) For all $\left(\boldsymbol{u}^{\mathbf{0}}, \boldsymbol{u}^{\mathbf{1}}, \boldsymbol{f}\right) \in \boldsymbol{V} \times \boldsymbol{H} \times L^{2}(0, T ; \boldsymbol{H})$, there exists a unique weak solution $\boldsymbol{u} \in C(0, T ; \boldsymbol{V}) \cap C^{1}(0, T ; \boldsymbol{H})$ that satisfies the variational problem

$$
<\boldsymbol{u}^{\prime \prime}, \boldsymbol{v}>_{\boldsymbol{V}^{\prime}, \boldsymbol{V}}+\int_{\omega} b^{\varepsilon}(\boldsymbol{u}, \boldsymbol{v}) d \xi=\int_{\omega} \boldsymbol{f} \cdot \boldsymbol{v} d \xi, \quad \text { a.e.t } \in(0, T), \forall \boldsymbol{v} \in \boldsymbol{V} .
$$

Moreover, the mapping $\left(\boldsymbol{u}^{\mathbf{0}}, \boldsymbol{u}^{\mathbf{1}}, \boldsymbol{f}\right) \rightarrow\left(\boldsymbol{u}(t), \boldsymbol{u}^{\prime}(t)\right)$ is continuous :

$$
\exists C>0 ;\|\boldsymbol{u}(t)\|_{\boldsymbol{V}}^{2}+\left\|\boldsymbol{u}^{\prime}(t)\right\|_{\boldsymbol{H}}^{2} \leq C\left(\left\|\boldsymbol{u}^{\mathbf{0}}\right\|_{\boldsymbol{V}}^{2}+\left|\boldsymbol{u}^{\mathbf{1}}\left\|_{\boldsymbol{H}}^{2}+\int_{0}^{t} \mid \boldsymbol{f}\right\|_{\boldsymbol{H}}^{2} d s\right), t \in(0, T) .\right.
$$

(ii) For all $\left(\boldsymbol{u}^{\mathbf{0}}, \boldsymbol{u}^{\mathbf{1}}, \boldsymbol{f}\right) \in \boldsymbol{E} \times \boldsymbol{V} \times L^{2}(0, T ; \boldsymbol{V})$, there exists a unique strong solution $\boldsymbol{u} \in$ $C(0, T ; \boldsymbol{E}) \cap C^{1}(0, T ; \boldsymbol{V})$ and

$$
\exists C>0:\|\boldsymbol{u}(t)\|_{\boldsymbol{E}}^{2}+\left\|\boldsymbol{u}^{\prime}(t)\right\|_{\boldsymbol{V}}^{2} \leq C\left(\left\|\boldsymbol{u}^{\mathbf{0}}\right\|_{\boldsymbol{E}}^{2}+\left\|\boldsymbol{u}^{\mathbf{1}}\right\|_{\boldsymbol{V}}^{2}+\int_{0}^{t}\|\boldsymbol{f}\|_{\boldsymbol{V}}^{2} d s\right), t \in(0, T) .
$$

When $\boldsymbol{f}=\mathbf{0}$, we denote by $E$ the "natural" energy of the arch

$$
E(t, \boldsymbol{u})=\frac{1}{2} \int_{\omega}\left(\boldsymbol{u}^{\prime} \cdot \boldsymbol{u}^{\prime}+b^{\varepsilon}(\boldsymbol{u}, \boldsymbol{u})\right) d \xi
$$

LEMMA 3.2 When $\boldsymbol{f}=\mathbf{0}$, the energy of the weak solution to (8) is constant in time: $E(t)=$ $E(0)$, for all $t \in(0, T)$.

Proof. First, when $\boldsymbol{u}$ is the strong solution to (8), multiplying (8) by $\boldsymbol{u}^{\prime}$ and integrating over $\omega$, we have $\int_{\omega}\left(\boldsymbol{u}^{\prime \prime}+\boldsymbol{A}^{\varepsilon} \boldsymbol{u}\right) \cdot \boldsymbol{u}^{\prime} d \xi=1 / 2 d / d t \int_{\omega}\left(\boldsymbol{u}^{\prime} \cdot \boldsymbol{u}^{\prime}+\boldsymbol{A}^{\varepsilon} \boldsymbol{u} \cdot \boldsymbol{u}\right) d \xi=1 / 2 d / d t \int_{\omega}\left(\boldsymbol{u}^{\prime} \cdot \boldsymbol{u}^{\prime}+\right.$ $\left.b^{\varepsilon}(\boldsymbol{u}, \boldsymbol{u})\right) d \xi$ using the step (ii) of Lemma 2.1. Secondly, when $\boldsymbol{u}$ is a weak solution associated with initial conditions $\left(\boldsymbol{u}^{\mathbf{0}}, \boldsymbol{u}^{\mathbf{1}}\right) \in \boldsymbol{V} \times \boldsymbol{H}$, we get the result by a density argument: let $\left(\boldsymbol{u}_{\boldsymbol{k}}^{\mathbf{0}}, \boldsymbol{u}_{\boldsymbol{k}}^{\mathbf{1}}\right)_{(k>0)} \in \boldsymbol{E} \times \boldsymbol{V}$ be a sequence of Cauchy conditions and let $\boldsymbol{u}_{\boldsymbol{k}}$ be the associated strong solution such that $\left(\boldsymbol{u}_{\boldsymbol{k}}^{\mathbf{0}}, \boldsymbol{u}_{\boldsymbol{k}}^{\mathbf{1}}\right) \rightarrow\left(\boldsymbol{u}^{\mathbf{0}}, \boldsymbol{u}^{\mathbf{1}}\right)$ in $\boldsymbol{V} \times \boldsymbol{H}$ as $k \rightarrow \infty$. From the continuous dependence of the solution with respect to the initial data, $\boldsymbol{u}_{\boldsymbol{k}} \rightarrow \boldsymbol{u}$ in $C(0, T ; \boldsymbol{V}) \cap C^{1}(0, T ; \boldsymbol{H})$. Hence, $E_{k}\left(t, \boldsymbol{u}_{\boldsymbol{k}}\right)=1 / 2\left(\left|\boldsymbol{u}_{\boldsymbol{k}}^{\prime}\right|_{\boldsymbol{H}}^{2}+\left|\boldsymbol{u}_{\boldsymbol{k}}\right|_{\boldsymbol{V}}^{2}\right) \rightarrow E(t, \boldsymbol{u})=1 / 2\left(\left|\boldsymbol{u}^{\prime}\right|_{\boldsymbol{H}}^{2}+|\boldsymbol{u}|_{\boldsymbol{V}}^{2}\right)$ as $k \rightarrow \infty$.

In the sequel, we take $\boldsymbol{f}=0$ and note

$$
E_{0}(\boldsymbol{u})=E(t=0, \boldsymbol{u})=\frac{1}{2} \int_{\omega}\left(\boldsymbol{u}^{\mathbf{1}} \cdot \boldsymbol{u}^{\mathbf{1}}+b^{\varepsilon}\left(\boldsymbol{u}^{\mathbf{0}}, \boldsymbol{u}^{\mathbf{0}}\right)\right) d \xi
$$

\subsection{Observability and direct inequalities on the homogeneous prob- lem}

In view of the null controllability, we establish in this section that for any $T$ large enough, $r^{-1}$ small enough and any initial data $\left(\boldsymbol{u}^{\mathbf{0}}, \boldsymbol{u}^{\mathbf{1}}\right) \in \boldsymbol{V} \times \boldsymbol{H}$, there exist two positive constants 
$C_{1}$ and $C_{2}$ such that

$$
C_{2} E_{0}(\boldsymbol{u}) \leq \int_{0}^{T}\left[b^{\varepsilon}(\boldsymbol{u}, \boldsymbol{u})\right]_{0}^{1} d t \leq C_{1} E_{0}(\boldsymbol{u})
$$

Those inequalities, obtained using the multiplier technic, show that $\left(\int_{0}^{T}\left[b^{\varepsilon}(\boldsymbol{u}, \boldsymbol{u})\right]_{0}^{1} d t\right)^{1 / 2}$ defines a norm on the set of initial data. The left part of (9) is called the observability inequality associated with the adjoint system (8) and allows to show that if $u$ solution of (8) is such that $u \equiv 0$ on $\Sigma_{T}$, then $u \equiv 0$ in $Q_{T}$. In that sense, the whole solution is observed from the boundary. This inequality requires that the time be large enough, due the finite speed of propagation of the solution along $\omega$. The right part of (9), easier to obtain, is called the direct inequality, and does not require any condition on $T$. The right inequality is also referred as the hidden inequality because implies extra regularity on the boundary terms.

\subsubsection{Some technical lemma}

The multiplier method is based on the multiplication of the conservative system (8) by suitable functions and allows to express the solution $u$ on $Q_{T}$ in term of the solution on $\Sigma_{T}$. For the sake of clarity, we collect in this section such computations leading to Proposition 3.1 .

Lemma 3.3 Let $\boldsymbol{u}$ be the strong solution of (8) and $q \in C^{1}(\bar{\omega})$. We have

$$
\int_{Q_{T}} \boldsymbol{u}^{\prime \prime} \cdot q \boldsymbol{u}, 1, d \xi d t=\frac{1}{2} \int_{Q_{T}} q_{, 1} \boldsymbol{u}^{\prime} \cdot \boldsymbol{u}^{\prime} d \xi d t+\int_{\omega}\left[\boldsymbol{u}^{\prime} \cdot q \boldsymbol{u}_{, 1}\right]_{0}^{T} d \xi .
$$

Proof. Integrating by part, we have

$$
\begin{aligned}
\int_{Q_{T}} \boldsymbol{u}^{\prime \prime} \cdot q \boldsymbol{u}_{, 1} d \xi d t & =-\int_{Q_{T}} \boldsymbol{u}^{\prime} \cdot q \boldsymbol{u}^{\prime}{ }_{, 1} d \xi d t+\int_{\omega}\left[\boldsymbol{u}^{\prime} \cdot q \boldsymbol{u}_{, 1}\right]_{0}^{T} d \xi \\
& =-\frac{1}{2} \int_{Q_{T}} q\left(\boldsymbol{u}^{\prime} \cdot \boldsymbol{u}^{\prime}\right)_{, 1} d \xi d t+\int_{\omega}\left[\boldsymbol{u}^{\prime} \cdot q \boldsymbol{u}_{, 1}\right]_{0}^{T} d \xi \\
& =\frac{1}{2} \int_{Q_{T}} q, \boldsymbol{u}^{\prime} \cdot \boldsymbol{u}^{\prime} d \xi d t-\frac{1}{2} \int_{0}^{T}\left[q \boldsymbol{u}^{\prime} \cdot \boldsymbol{u}^{\prime}\right]_{0}^{1} d t+\int_{\omega}\left[\boldsymbol{u}^{\prime} \cdot q \boldsymbol{u}_{, 1}\right]_{0}^{T} d \xi
\end{aligned}
$$

and the result follows since $\boldsymbol{u}=0$ on $\Sigma_{T}=\partial \omega \times(0, T)$.

We also have the following relations.

Lemma 3.4 For all $q \in C^{2}(\bar{\omega})$ and $\boldsymbol{v} \in C^{2}(\bar{\omega}) \times C^{3}(\bar{\omega})$ we have

$$
\gamma(q \boldsymbol{v}, 1)=q \gamma(\boldsymbol{v})_{, 1}+g_{M}(q, \boldsymbol{v}), \quad \rho\left(q \boldsymbol{v}_{, 1}\right)=q \rho(\boldsymbol{v})_{, 1}+g_{F}(q, \boldsymbol{v})
$$

with

$$
g_{M}(q, \boldsymbol{v})=q_{, 1} v_{1,1}, \quad g_{F}(q, \boldsymbol{v})=q_{, 11} v_{3,1}+2 q_{, 1} v_{3,11}-2 r^{-1} q_{, 1} v_{1,1}
$$


Proof. From Definition 2.1, we write for all $q \in C^{1}(\bar{\omega})$ and $\boldsymbol{v} \in C^{2}(\bar{\omega}) \times C^{1}(\bar{\omega})$ that

$$
\begin{aligned}
\gamma\left(q \boldsymbol{v}_{, 1}\right) & =\left(q v_{1,1}\right)_{, 1}+r^{-1} q v_{3,1}=q_{, 1} v_{1,1}+q v_{1,11}+r^{-1} q v_{3,1} \\
& =q\left(v_{1,1}+r^{-1} v_{3}\right)_{, 1}+q, 1 v_{1,1}=q \gamma(\boldsymbol{v})_{, 1}+g_{M}(q, \boldsymbol{v})
\end{aligned}
$$

Similarly, for all $q \in C^{2}(\bar{\omega})$ and $\boldsymbol{v} \in C^{2}(\bar{\omega}) \times C^{3}(\bar{\omega})$, we write

$$
\begin{aligned}
& \rho\left(q \boldsymbol{v}_{, 1}\right)=\left(q v_{3,1}\right)_{, 11}-2 r^{-1}\left(q v_{1,1}\right)_{, 1}-r^{-2} q v_{3,1} \\
&=q, 11 v_{3,1}+q v_{3,111}+2 q_{, 1} v_{3,11}-2 r^{-1}\left(q_{, 1} v_{1,1}+q v_{1,11}\right)-r^{-2} q v_{3,1} \\
&=q\left(v_{3,11}-2 r^{-1} v_{1,1}-r^{-2} v_{3}\right)_{, 1}+q, 11 \\
& v_{3,1}+2 q_{, 1} v_{3,11}-2 r^{-1} q_{, 1} v_{1,1} \\
&=q \rho(\boldsymbol{v})_{, 1}+g_{F}(q, \boldsymbol{v}) .
\end{aligned}
$$

Lemma 3.4 then implies the following one.

Lemma 3.5 For all $q \in C^{2}(\bar{w})$ and $\boldsymbol{v} \in H^{3}(\omega) \times H^{4}(\omega)$, we have

$$
\int_{\omega} b^{\varepsilon}(\boldsymbol{v}, q \boldsymbol{v}, 1) d \xi=-\frac{1}{2} \int_{\omega} q, 1 b^{\varepsilon}(\boldsymbol{v}, \boldsymbol{v}) d \xi+\frac{1}{2}\left[q b^{\varepsilon}(\boldsymbol{v}, \boldsymbol{v})\right]_{0}^{1}+\int_{\omega} g^{\varepsilon}(q, \boldsymbol{v}) d \xi
$$

where

$$
g^{\varepsilon}(q, \boldsymbol{v})=\gamma(\boldsymbol{v}) g_{M}(q, \boldsymbol{v})+\frac{\varepsilon^{2}}{3} \rho(\boldsymbol{v}) g_{F}(q, \boldsymbol{v}) .
$$

Proof. From the definition of $b_{M}$ (see Definition 2.1) and using Lemma 3.4, we write

$$
\begin{aligned}
\int_{\omega} b_{M}\left(\boldsymbol{v}, q \boldsymbol{v}_{, 1}\right) d \xi & =\int_{\omega} \gamma(\boldsymbol{v}) \gamma(q \boldsymbol{v}, 1) d \xi=\int_{\omega} \gamma(\boldsymbol{v})\left(q \gamma(\boldsymbol{v})_{, 1}+g_{M}(q, \boldsymbol{v})\right) d \xi \\
& =\frac{1}{2} \int_{\omega} q b_{M}(\boldsymbol{v}, \boldsymbol{v})_{, 1} d \xi+\int_{\omega} \gamma(\boldsymbol{v}) g_{M}(q, \boldsymbol{v}) d \xi \\
& =-\frac{1}{2} \int_{\omega} q_{, 1} b_{M}(\boldsymbol{v}, \boldsymbol{v}) d \xi+\frac{1}{2}\left[q b_{M}(\boldsymbol{v}, \boldsymbol{v})\right]_{0}^{1}+\int_{\omega} \gamma(\boldsymbol{v}) g_{M}(q, \boldsymbol{v}) d \xi
\end{aligned}
$$

A similar computation for the term $\int_{\omega} b_{F}(\boldsymbol{v}, q \boldsymbol{v}, 1) d \xi$ then leads to the result.

Lemma 3.6 For all $\left(\boldsymbol{u}^{\mathbf{0}}, \boldsymbol{u}^{\mathbf{1}}\right) \in \boldsymbol{E} \times \boldsymbol{V}$ and all $q \in C^{2}(\bar{w})$, we have

$$
\int_{\omega} \boldsymbol{A}^{\varepsilon} \boldsymbol{u} \cdot q \boldsymbol{u}, 1 d \xi=\int_{\omega} b^{\varepsilon}(\boldsymbol{u}, q \boldsymbol{u}, 1) d \xi-\left[q b^{\varepsilon}(\boldsymbol{u}, \boldsymbol{u})\right]_{0}^{1} .
$$

Proof. Since $\boldsymbol{u}$ is a strong solution to (8), we use the step (i) of Lemma 2.1 with $\boldsymbol{v}=q \boldsymbol{u}, 1$. We write

$$
\int_{\omega} \boldsymbol{A}_{M} \boldsymbol{u} \cdot q \boldsymbol{u}, 1 d \xi=\int_{\omega} b_{M}\left(\boldsymbol{u}, q \boldsymbol{u}_{, 1}\right) d \xi-\left[\gamma(\boldsymbol{u}) q u_{1,1}\right]_{0}^{1} .
$$

Since $u_{3}=0$ on $\partial \omega \times(0, T)$, the last integral is

$$
-\left[\gamma(\boldsymbol{u}) q\left(u_{1,1}+r^{-1} u_{3}\right)\right]_{0}^{1}=-[\gamma(\boldsymbol{u}) \gamma(\boldsymbol{u}) q]_{0}^{1}=-\left[b_{M}(\boldsymbol{u}, \boldsymbol{u}) q\right]_{0}^{1} .
$$


Similarly,

$$
\int_{\omega} \boldsymbol{A}_{F} \boldsymbol{u} \cdot q \boldsymbol{u}_{, 1} d \xi=\int_{\omega} b_{F}\left(\boldsymbol{u}, q \boldsymbol{u}_{, 1}\right) d \xi+\left[q\left(\rho(\boldsymbol{u})_{, 1} u_{3,1}-\rho(\boldsymbol{u}) u_{3,11}+2 r^{-1} u_{1,1}\right)\right]_{0}^{1} .
$$

Once again, since $u_{3}=u_{3,1}=0$ on $\partial \omega$, the last term is $\left[q \rho(\boldsymbol{u})\left(-u_{3,11}+2 r^{-1} u_{1,1}+r^{-2} u_{3}\right)\right]_{0}^{1}=$ $-[q \rho(\boldsymbol{u}) \rho(\boldsymbol{u})]_{0}^{1}=-\left[q b_{F}(\boldsymbol{u}, \boldsymbol{u})\right]_{0}^{1}$. The result follows from $b^{\varepsilon}(\boldsymbol{u}, \boldsymbol{u})=b_{M}(\boldsymbol{u}, \boldsymbol{u})+\varepsilon^{2} / 3 b_{F}(\boldsymbol{u}, \boldsymbol{u})$.

These lemmata lead to the following result.

Proposition 3.1 Let $q \in C^{2}(\bar{\omega})$. For all $\left(\boldsymbol{u}^{\mathbf{0}}, \boldsymbol{u}^{\mathbf{1}}\right) \in \boldsymbol{V} \times \boldsymbol{H}$, the weak solution to (8) satisfies the identity

$$
\begin{aligned}
\frac{1}{2} \int_{0}^{T}\left[q b^{\varepsilon}(\boldsymbol{u}, \boldsymbol{u})\right]_{0}^{1} d t= & \frac{1}{2} \int_{Q_{T}} q_{, 1}\left(\boldsymbol{u}^{\prime} \cdot \boldsymbol{u}^{\prime}-b^{\varepsilon}(\boldsymbol{u}, \boldsymbol{u})\right) d \xi d t+\int_{\omega}\left[\boldsymbol{u}^{\prime} \cdot q \boldsymbol{u}_{, 1}\right]_{0}^{T} d \xi \\
& +\int_{Q_{T}} g^{\varepsilon}(q, \boldsymbol{u}) d \xi d t .
\end{aligned}
$$

Proof. As in Proposition We first consider the strong solution associated with $\left(\boldsymbol{u}^{\mathbf{0}}, \boldsymbol{u}^{\mathbf{1}}\right) \in$ $\boldsymbol{E} \times \boldsymbol{V}$. Multiplying (8) by the multiplier $q \boldsymbol{u}_{, 1}$ and integrating over $Q_{T}$, we obtain $\int_{Q_{T}}\left(\boldsymbol{u}^{\prime \prime}\right.$. $\left.q \boldsymbol{u}_{, 1}+\boldsymbol{A}^{\boldsymbol{\varepsilon}} \boldsymbol{u} \cdot q \boldsymbol{u}_{, 1}\right) d \xi=0$ and the result follows using Lemmas 3.3,3.5 and 3.6. For weak solution of (8) with the data $\left(\boldsymbol{u}^{\mathbf{0}}, \boldsymbol{u}^{\mathbf{1}}\right) \in \boldsymbol{V} \times \boldsymbol{H}$, the result is obtained using a classical density argument. (see [18] for the wave and beam equation and [20] for the general situation of shell operator.)

\subsubsection{Direct and inverse inequality}

We are now in a position to prove the right inequality of (9), the so-called direct (or hiddenregularity) inequality.

TheOREM 3.1 Let $\varepsilon>0$. There exists a constant $C_{1}$, which depends only on $\omega$ and on the map $\boldsymbol{\varphi}$ such that for all $\left(\boldsymbol{u}^{\mathbf{0}}, \boldsymbol{u}^{\mathbf{1}}\right) \in \boldsymbol{V} \times \boldsymbol{H}$, the weak solution to (8) satisfies the inequality

$$
\int_{0}^{T}\left[b^{\varepsilon}(\boldsymbol{u}, \boldsymbol{u})\right]_{0}^{1} d t \leq C_{1}(1+T) E_{0}(\boldsymbol{u}) .
$$

Therefore, the linear mapping $\left(\boldsymbol{u}^{\mathbf{0}}, \boldsymbol{u}^{\mathbf{1}}\right) \in \boldsymbol{V} \times \boldsymbol{H} \rightarrow b^{\varepsilon}(\boldsymbol{u}, \boldsymbol{u}) \in L^{1}\left(\Sigma_{T}\right)$ is continuous.

Proof. Let us take $q \in C^{2}(\bar{\omega})$ such that $q(0)=-1$ and $q(1)=1$. It follows from (10) that

$$
\begin{aligned}
\int_{0}^{T}\left[b^{\varepsilon}(\boldsymbol{u}, \boldsymbol{u})\right]_{0}^{1} d t= & \int_{Q_{T}} q_{, 1}\left(\boldsymbol{u}^{\prime} \cdot \boldsymbol{u}^{\prime}-b^{\varepsilon}(\boldsymbol{u}, \boldsymbol{u})\right) d \xi d t+2 \int_{\omega}\left[\boldsymbol{u}^{\prime} \cdot q \boldsymbol{u}_{, 1}\right]_{0}^{T} d \xi \\
& +2 \int_{Q_{T}} g^{\varepsilon}(q, \boldsymbol{u}) d \xi d t .
\end{aligned}
$$

The first integral is lower than $\left\|q_{, 1}\right\|_{L^{\infty}(\omega)} T E_{0}(\boldsymbol{u})$. Similarly, using that $\left(\int_{\omega} b^{\varepsilon}(\boldsymbol{u}, \boldsymbol{u}) d \xi\right)^{1 / 2}$ is a norm equivalent to $\|\boldsymbol{u}\|_{\boldsymbol{V}}$, the third term is lower than $2 C T \int_{\omega} b^{\varepsilon}(\boldsymbol{u}, \boldsymbol{u}) d \xi \leq 2 C T E_{0}(\boldsymbol{u})$ for some $C>0$. At last, the second term is less than $4 C E_{0}(\boldsymbol{u})$. The majoration follows. 
The left inequality in (9) - the observability inequality - is obtained with the new multiplier $p=2\left(\xi-\xi_{0}\right), \xi_{0} \in \mathbb{R}$, in this case Proposition 3.1 takes the following form.

Proposition 3.2 Let $p=2\left(\xi-\xi_{0}\right), \xi_{0} \in \mathbb{R}$. For all $\left(\boldsymbol{u}^{\mathbf{0}}, \boldsymbol{u}^{\mathbf{1}}\right) \in \boldsymbol{V} \times \boldsymbol{H}$, the weak solution to (8) satisfies the identity

$$
\begin{aligned}
\frac{1}{2} \int_{0}^{T}\left[p b^{\varepsilon}(\boldsymbol{u}, \boldsymbol{u})\right]_{0}^{1} d t= & T E_{0}(\boldsymbol{u})+\int_{Q_{T}}\left(\gamma(\boldsymbol{u}) \gamma(\boldsymbol{u})+\varepsilon^{2} \rho(\boldsymbol{u}) \rho(\boldsymbol{u})\right) d \xi d t \\
& +\int_{Q_{T}} h^{\varepsilon}(\boldsymbol{u}) d \xi+\int_{\omega}\left[\boldsymbol{u}^{\prime} \cdot\left(p \boldsymbol{u}_{, 1}+\frac{\boldsymbol{u}}{2}\right)\right]_{0}^{T} d \xi
\end{aligned}
$$

Proof. As in Proposition 3.1, the identity is first established for Cauchy conditions $\left(\boldsymbol{u}^{\mathbf{0}}, \boldsymbol{u}^{\mathbf{1}}\right) \in \boldsymbol{E} \times \boldsymbol{V}$ corresponding to a strong solution and by the same density argument we show that it is still valid for $\left(\boldsymbol{u}^{\mathbf{0}}, \boldsymbol{u}^{\mathbf{1}}\right) \in \boldsymbol{V} \times \boldsymbol{H}$.

Hence, Lemma 3.3 reads

$$
\int_{Q_{T}} \boldsymbol{u}^{\prime \prime} \cdot p \boldsymbol{u}_{, 1} d \xi d t=\frac{1}{2} \int_{Q_{T}} 2 \boldsymbol{u}^{\prime} \cdot \boldsymbol{u}^{\prime} d \xi d t+\int_{\omega}\left[\boldsymbol{u}^{\prime} \cdot p \boldsymbol{u}_{, 1}\right]_{0}^{T} d \xi
$$

From the relation

$$
\int_{Q_{T}} \boldsymbol{u}^{\prime} \cdot \boldsymbol{u}^{\prime} d \xi d t=\int_{\omega}\left[\boldsymbol{u}^{\prime} \cdot \boldsymbol{u}\right]_{0}^{T} d \xi+\int_{Q_{T}} b^{\varepsilon}(\boldsymbol{u}, \boldsymbol{u}) d \xi d t
$$

we obtain that

$$
\begin{aligned}
\int_{Q_{T}} \boldsymbol{u}^{\prime \prime} \cdot p \boldsymbol{u}_{, 1} d \xi d t= & \frac{1}{2} \int_{Q_{T}} \boldsymbol{u}^{\prime} \cdot \boldsymbol{u}^{\prime} d \xi d t+\frac{1}{2} \int_{Q_{T}} b^{\varepsilon}(\boldsymbol{u}, \boldsymbol{u}) d \xi d t+\int_{\omega}\left[\boldsymbol{u}^{\prime} \cdot\left(p \boldsymbol{u}_{, 1}+\frac{\boldsymbol{u}}{2}\right)\right]_{0}^{T} d \xi \\
& =T E_{0}(\boldsymbol{u})+\int_{\omega}\left[\boldsymbol{u}^{\prime} \cdot\left(p \boldsymbol{u}_{, 1}+\frac{\boldsymbol{u}}{2}\right)\right]_{0}^{T} d \xi
\end{aligned}
$$

Lemma 3.4 now reads

$$
\begin{aligned}
& \gamma\left(p \boldsymbol{v}_{, 1}\right)=p \gamma(\boldsymbol{v})_{, 1}+2 v_{1,1}=p \gamma(\boldsymbol{v})_{, 1}+2 \gamma(\boldsymbol{v})+h_{M}(\boldsymbol{v}) \\
& \rho\left(p \boldsymbol{v}_{, 1}\right)=p \rho(\boldsymbol{v})_{, 1}+4 v_{3,11}-4 r^{-1} v_{1,1} \equiv p \rho(\boldsymbol{v})_{, 1}+4 \rho(\boldsymbol{v})+h_{F}(\boldsymbol{v})
\end{aligned}
$$

where $h_{M}(\boldsymbol{v})=-2 r^{-1} v_{3}$ and $h_{F}(\boldsymbol{v})=4 r^{-1} \gamma(\boldsymbol{v})$. At last Lemma 3.5 becomes

$$
\int_{\omega} b^{\varepsilon}\left(\boldsymbol{u}, p \boldsymbol{u}_{, 1}\right) d \xi=\frac{1}{2}\left[p b^{\varepsilon}(\boldsymbol{u}, \boldsymbol{u})\right]_{0}^{1}+\int_{\omega}\left(\gamma(\boldsymbol{u}) \gamma(\boldsymbol{u})+\varepsilon^{2} \rho(\boldsymbol{u}) \rho(\boldsymbol{u})\right) d \xi+\int_{\omega} h^{\varepsilon}(\boldsymbol{u}) d \xi
$$

where

$$
h^{\varepsilon}(\boldsymbol{u})=\gamma(\boldsymbol{u}) h_{M}(\boldsymbol{u})+\frac{\varepsilon^{2}}{3} \rho(\boldsymbol{u}) h_{F}(\boldsymbol{u}) .
$$

Summarizing these equalities yields the result.

Before showing the left part of (9), we need the following intermediate result : 
Proposition 3.3 Let $p=2\left(\xi-\xi_{0}\right), \xi_{0} \in \mathbb{R}, \varepsilon>0$ and $r^{-1}>0$. There exist a constant $C>0$ which depends on $\omega, \varepsilon$ and on the map $\varphi$ and a constant $D$ which depends on $\varepsilon$ such that for all $\left(\boldsymbol{u}^{\mathbf{0}}, \boldsymbol{u}^{\mathbf{1}}\right) \in \boldsymbol{V} \times \boldsymbol{H}$, the weak solution to (8) satisfies the inequality

$$
\frac{1}{2} \int_{0}^{T}\left[p b^{\varepsilon}(\boldsymbol{u}, \boldsymbol{u})\right]_{0}^{1} d t \geq\left(T\left(1-r^{-1} D(\varepsilon)\right)-2 C\right) E_{0}(\boldsymbol{u}) .
$$

Proof. We first prove that there exists a constant $C$ such that

$$
\int_{\omega} \boldsymbol{u}^{\prime} \cdot\left(p \boldsymbol{u}_{, \mathbf{1}}+\frac{\boldsymbol{u}}{2}\right) d \xi \leq C E_{0}(\boldsymbol{u}), \quad \forall t \geq 0
$$

For any constant $B>0$, we have

$$
2\left|\int_{\omega} \boldsymbol{u}^{\prime} \cdot\left(p \boldsymbol{u}_{, 1}+\frac{\boldsymbol{u}}{2}\right) d \xi\right| \leq \int_{\omega}\left(B \boldsymbol{u}^{\prime} \cdot \boldsymbol{u}^{\prime}+\frac{1}{B}\left(p \boldsymbol{u}_{, 1}+\frac{\boldsymbol{u}}{2}\right) \cdot\left(p \boldsymbol{u}_{, 1}+\frac{\boldsymbol{u}}{2}\right)\right) d \xi .
$$

Using that $\int_{\omega} p \boldsymbol{u}_{, 1} \cdot \boldsymbol{u} d \xi=-\int_{\omega} \boldsymbol{u} \cdot \boldsymbol{u} d \xi$, we obtain

$$
\int_{\omega}\left|p \boldsymbol{u}_{, 1}+\frac{\boldsymbol{u}}{2}\right|^{2} d \xi \leq \int_{\omega} p \boldsymbol{u}_{, 1} \cdot p \boldsymbol{u}_{, 1} d \xi
$$

Moreover, from Poincaré's inequality, there exists a constant $C_{K}$ such that

$$
\int_{\omega}\left(\boldsymbol{u}_{, 1} \cdot \boldsymbol{u}_{, 1}+\boldsymbol{u} \cdot \boldsymbol{u}\right) d \xi \leq C_{K} \int_{\omega} b^{\varepsilon}(\boldsymbol{u}, \boldsymbol{u}) d \xi
$$

Hence,

$$
\left|\int_{\omega} \boldsymbol{u}^{\prime} \cdot\left(p \boldsymbol{u}_{, 1}+\frac{\boldsymbol{u}}{2}\right) d \xi\right| \leq \frac{1}{2} \max \left(B, C_{K} \frac{\|p\|_{L^{\infty}(\omega)}^{2}}{B}\right) E_{0}(\boldsymbol{u})
$$

(12) then follows with $B=\sqrt{C_{K}}\|p\|_{L^{\infty}(\omega)}$ and $C=B / 2$. From Proposition 3.2, the intermediate result is therefore that

$$
\frac{1}{2} \int_{0}^{T}\left[p b^{\varepsilon}(\boldsymbol{u}, \boldsymbol{u})\right]_{0}^{1} d t \geq(T-2 C) E_{0}(\boldsymbol{u})+\int_{Q_{T}} h^{\varepsilon}(\boldsymbol{u}) d \xi d t .
$$

From $h^{\varepsilon}(\boldsymbol{u})=r^{-1} \gamma(\boldsymbol{u})\left(-2 u_{3}+4 \frac{\varepsilon^{2}}{3} \rho(\boldsymbol{u})\right)$ we first notice that the last term vanishes for $r^{-1}=0$ (corresponding to a flat arch) and then (11) follows with $D=0$. For $r^{-1}>0$, using the equivalence of the norm in $\boldsymbol{V}$ and the elastic energy norm $\left(\int_{\omega} b^{\varepsilon}(\boldsymbol{u}, \boldsymbol{u}) d \xi\right)^{1 / 2}$, there exists a constant $D(\varepsilon)$ independent of $r$ such that

$$
\left|\int_{\omega} h^{\varepsilon}(\boldsymbol{u}) d \xi\right| \leq r^{-1} D(\varepsilon) \int_{\omega} b^{\varepsilon}(\boldsymbol{u}, \boldsymbol{u}) d \xi
$$

(11) then follows. 
Let us now take $\xi_{0} \in \omega$ so that $p$ defined by $p(\xi)=2\left(\xi-\xi_{0}\right)$ satisfies $p(0)<0$ and $p(1)>0$. Then, defining

$$
T^{\star}\left(r^{-1}, \varepsilon\right)=\frac{2 C}{1-r^{-1} D(\varepsilon)}
$$

and assuming $r$ large enough so that $1-r^{-1} D(\varepsilon)>0$, we deduce from (11) the following result:

TheOREm 3.2 Let $\varepsilon>0$ and $r^{-1}<1 / D(\varepsilon)$. For all $T>T^{\star}\left(r^{-1}, \varepsilon\right)$, there exists a constant $C_{2}=\left(1-r^{-1} D(\varepsilon)\right)$ such that for all $\left(\boldsymbol{u}^{\mathbf{0}}, \boldsymbol{u}^{\mathbf{1}}\right) \in \boldsymbol{V} \times \boldsymbol{H}$, the weak solution to (8) satisfies the inequality

$$
\int_{0}^{T}\left[b^{\varepsilon}(\boldsymbol{u}, \boldsymbol{u})\right]_{0}^{1} d t \geq C_{2}\left(r^{-1}, \varepsilon\right)\left(T-T^{\star}\right) E_{0}(\boldsymbol{u}) .
$$

We then conclude from Theorem 3.1 and Theorem 3.2 that for all $\left(\boldsymbol{u}^{\mathbf{0}}, \boldsymbol{u}^{\mathbf{1}}\right) \in \boldsymbol{V} \times \boldsymbol{H}$, if $r^{-1}<1 / D(\varepsilon)$ and $T>T^{\star}$, then the semi-norm $\left(\int_{0}^{T}\left[b^{\varepsilon}(\boldsymbol{u}, \boldsymbol{u})\right]_{0}^{1} d t\right)^{1 / 2}$ is a norm over $\boldsymbol{V} \times \boldsymbol{H}$. Similarly, the observability from the right extremity $\xi=1$ is obtained with the choice $\xi_{0}=0$ in $p$.

\section{The control problem}

Let $\boldsymbol{V}^{\prime}$ the dual space of $\boldsymbol{V}$ defined by $\boldsymbol{V}^{\prime}=H^{-1}(\omega) \times H^{-2}(\omega)$. We show in this section that for any $\left(\boldsymbol{y}^{\mathbf{0}}, \boldsymbol{y}^{\mathbf{1}}\right)$ in $\boldsymbol{H} \times \boldsymbol{V}^{\prime}$, there exists a control function $\boldsymbol{v} \in\left(L^{2}\left(\Sigma_{T}\right)\right)^{2}$ such that the solution $\boldsymbol{y}$ of

$$
\left\{\begin{array}{lr}
\boldsymbol{y}^{\prime \prime}+\boldsymbol{A}^{\boldsymbol{\varepsilon}} \boldsymbol{y}=\mathbf{0} & \text { in } Q_{T}, \\
y_{1}=v_{1}, y_{3}=0, \partial_{\nu} y_{3}=v_{3} & \text { on } \Sigma_{T}, \\
\left(\boldsymbol{y}(\cdot, 0), \boldsymbol{y}^{\prime}(\cdot, 0)\right)=\left(\boldsymbol{y}^{\mathbf{0}}, \boldsymbol{y}^{\mathbf{1}}\right) & \text { in } \omega
\end{array}\right.
$$

is at rest at time $T$, i.e. satisfy (2). Following [20], we first establish an existence theorem for (13) and then construct the control $\boldsymbol{v}$.

Proposition 4.1 For any $\left(\boldsymbol{y}^{\mathbf{0}}, \boldsymbol{y}^{\mathbf{1}}, \boldsymbol{v}\right) \in \boldsymbol{H} \times \boldsymbol{V}^{\mathbf{\prime}} \times\left(L^{2}\left(\Sigma_{T}\right)\right)^{2}$, there exists a unique solution $\boldsymbol{y} \in C(0, T ; \boldsymbol{H}) \cap C^{1}\left(0, T ; \boldsymbol{V}^{\prime}\right)$ and the mapping $\left(\boldsymbol{y}^{\mathbf{0}}, \boldsymbol{y}^{\mathbf{1}}, \boldsymbol{v}\right) \rightarrow\left(\boldsymbol{y}(t), \boldsymbol{y}^{\prime}(t)\right)$ is continuous:

$$
\exists C>0:\|\boldsymbol{y}(t)\|_{\boldsymbol{H}}^{2}+\left\|\boldsymbol{y}^{\prime}(t)\right\|_{\boldsymbol{V}^{\prime}}^{2} \leq C\left(\left\|\boldsymbol{y}^{\mathbf{0}}\right\|_{\boldsymbol{H}}^{2}+\left\|\boldsymbol{y}^{\mathbf{1}}\right\|_{\boldsymbol{V}^{\prime}}^{2}+\|\boldsymbol{v}\|_{\left(L^{2}\left(\Sigma_{T}\right)\right)^{2}}^{2}, \quad t \in(0, T)\right.
$$

PRoOF. We refer to a detailed proof for hyperbolic equation to [18] and to [20] for shell system. For clarity, let us simply precise how the estimate is obtained. Let us first define a weak solution of $(13)$. Let $\mathcal{D}(\omega)=C_{c}^{\infty}(\bar{\omega})$. For all $\left(\boldsymbol{u}^{\mathbf{0}}, \boldsymbol{u}^{\mathbf{1}}\right) \in \mathcal{D}(\omega) \times \mathcal{D}(\omega)$, let $\boldsymbol{u}$ be the unique solution of (8). Multiplying (13) by $\boldsymbol{u}$ and (8) by $\boldsymbol{y}$, and integrating over $Q_{T}$, we 
get, for all $t \in(0, T)$

$$
\begin{aligned}
\int_{\omega}\left(\boldsymbol{u}^{\prime}(t) \cdot \boldsymbol{y}(t)-\boldsymbol{u}(t) \cdot \boldsymbol{y}^{\prime}(t)\right) d \xi & =\int_{\omega}\left(\boldsymbol{u}^{\mathbf{1}} \cdot \boldsymbol{y}^{\mathbf{0}}-\boldsymbol{y}^{\mathbf{1}} \cdot \boldsymbol{u}^{\mathbf{0}}\right) d \xi+\int_{0}^{t} \int_{\omega}\left(A^{\varepsilon} \boldsymbol{y} \cdot \boldsymbol{u}-\boldsymbol{A}^{\varepsilon} \boldsymbol{u} \cdot \boldsymbol{y}\right) d t d \xi \\
& =\int_{\omega}\left(\boldsymbol{u}^{\mathbf{1}} \cdot \boldsymbol{y}^{\mathbf{0}}-\boldsymbol{y}^{\mathbf{1}} \cdot \boldsymbol{u}^{\mathbf{0}}\right) d \xi+\int_{0}^{t}\left[B^{\varepsilon}(\boldsymbol{u}, \boldsymbol{v})\right]_{0}^{1} d t
\end{aligned}
$$

where, from Lemma 2.1, the boundary operator $B^{\varepsilon}$ reads

$$
B^{\varepsilon}(\boldsymbol{u}, \boldsymbol{v})=\gamma(\boldsymbol{u}) v_{1}+\frac{\varepsilon^{2}}{3} \rho(\boldsymbol{u})\left(v_{3,1}-2 r^{-1} v_{1}\right)
$$

Noting $\langle\boldsymbol{u}, \boldsymbol{v}\rangle=\int_{\omega} \boldsymbol{u} \cdot \boldsymbol{v} d \xi$ the duality product between $\boldsymbol{H}$ and $\boldsymbol{H}^{\prime}(=\boldsymbol{H})$ or $\boldsymbol{V}$ and $\boldsymbol{V}^{\prime}$, the previous equality becomes

$$
<\boldsymbol{u}^{\prime}(t), \boldsymbol{y}(t)>-<\boldsymbol{u}(t), \boldsymbol{y}^{\prime}(t)>=<\boldsymbol{u}^{\mathbf{1}}, \boldsymbol{y}^{\mathbf{0}}>-<\boldsymbol{y}^{\mathbf{1}}, \boldsymbol{u}^{\mathbf{0}}>+\int_{0}^{t}\left[B^{\varepsilon}(\boldsymbol{u}, \boldsymbol{v})\right]_{0}^{1} d t
$$

Therefore, $\boldsymbol{y}$ is a weak solution to (13) if it satisfies (14). Now, according to Lemma 3.1, for $\left(\boldsymbol{u}^{\mathbf{0}}, \boldsymbol{u}^{\mathbf{1}}\right) \in \boldsymbol{V} \times \boldsymbol{H}$, there exists $C_{1}>0$ such that

$$
\|\boldsymbol{u}(t)\|_{\boldsymbol{V}}+\left\|\boldsymbol{u}^{\prime}(t)\right\|_{\boldsymbol{H}}+\left(\int_{0}^{t}\left[b^{\varepsilon}(\boldsymbol{u}(s), \boldsymbol{u}(s))\right]_{0}^{1} d s\right)^{1 / 2} \leq C_{1}\left(\left\|\boldsymbol{u}^{\mathbf{0}}\right\|_{\boldsymbol{V}}+\left\|\boldsymbol{u}^{\mathbf{1}}\right\|_{\boldsymbol{H}}\right) .
$$

Moreover, from the definition of $B^{\varepsilon}$, there exists a constant $C_{2}>0$ such that

$$
\int_{0}^{t}\left[B^{\varepsilon}(\boldsymbol{u}, \boldsymbol{v})\right]_{0}^{1} d s \leq C_{2}|\boldsymbol{v}|_{\left(L^{2}\left(\Sigma_{T}\right)\right)^{2}}\left(\int_{0}^{t}\left[b^{\varepsilon}(\boldsymbol{u}(s), \boldsymbol{u}(s)) d s\right]_{0}^{1}\right)^{1 / 2} .
$$

The estimate of Proposition 4.1 then follows from the relation (14). Finally, the regularity on $\boldsymbol{y}$ is obtained by density argument.

We can now apply the duality HUM method (see [18]) to get the following controllability result.

Theorem 4.1 (Null Controllability) Let $\varepsilon>0, r>D(\varepsilon)$ and $T>T^{\star}$. For any $\left(\boldsymbol{y}^{\mathbf{0}}, \boldsymbol{y}^{\mathbf{1}}\right) \in \boldsymbol{H} \times \boldsymbol{V}^{\prime}$, there exists a control $\boldsymbol{v}=\left(v_{1}, v_{3}\right) \in\left(L^{2}\left(\Sigma_{T}\right)\right)^{2}$ such that $\boldsymbol{y}(\cdot, T)=$ $\boldsymbol{y}^{\prime}(\cdot, T)=0$ in $\omega$.

Proof. We introduce the following backward system :

$$
\left\{\begin{array}{lr}
\boldsymbol{z}^{\prime \prime}+\boldsymbol{A}^{\varepsilon} \boldsymbol{z}=0 & \text { in } Q_{T} \\
z_{1}=\partial_{\nu} u_{1}, z_{3}=0, \partial_{\nu} z_{3}=\partial_{\nu \nu}^{2} u_{3} & \text { on } \Sigma_{T} \\
\left(\boldsymbol{z}(\cdot, T), \boldsymbol{z}^{\prime}(\cdot, T)\right)=(\mathbf{0}, \mathbf{0}) & \text { in } \omega .
\end{array}\right.
$$


From Proposition 4.1 and from the reversibility property of (15), there exists a unique solution $\boldsymbol{z} \in C(0, T ; \boldsymbol{H}) \cap C^{1}\left(0, T ; \boldsymbol{V}^{\prime}\right)$. Moreover, since

$$
\begin{aligned}
<\boldsymbol{z}^{\prime}(0), \boldsymbol{u}^{\mathbf{0}}>-< & \boldsymbol{u}^{\mathbf{1}}, \boldsymbol{z}(0)>=\int_{0}^{T}\left[\gamma(\boldsymbol{u}) z_{1}\right]_{0}^{1} d t \\
& +\frac{\varepsilon^{2}}{3} \int_{0}^{T}\left[\left(-\rho(\boldsymbol{u})_{, 1} z_{3}+\rho(\boldsymbol{u}) z_{3,1}-2 r^{-1} \rho(\boldsymbol{u}) z_{1}\right)\right]_{0}^{1} d t
\end{aligned}
$$

we get

$$
<\boldsymbol{z}^{\prime}(0), \boldsymbol{u}^{\mathbf{0}}>-<\boldsymbol{u}^{\mathbf{1}}, \boldsymbol{z}(0)>=\int_{0}^{T}\left[B^{\varepsilon}(\boldsymbol{u}, \boldsymbol{z})\right]_{0}^{1} d t=\int_{0}^{T}\left[b^{\varepsilon}(\boldsymbol{u}, \boldsymbol{u})\right]_{0}^{1} d t
$$

Then, as a consequence of (9), the linear mapping $\Lambda^{\varepsilon}:\left(\boldsymbol{u}^{\mathbf{0}}, \boldsymbol{u}^{\mathbf{1}}\right) \in \boldsymbol{V} \times \boldsymbol{H} \rightarrow\left(\boldsymbol{z}^{\prime}(0),-\boldsymbol{z}(0)\right) \in$ $\boldsymbol{V}^{\prime} \times \boldsymbol{H}$ verifies

$$
<\Lambda^{\varepsilon}\left(\boldsymbol{u}^{\mathbf{0}}, \boldsymbol{u}^{\mathbf{1}}\right),\left(\boldsymbol{u}^{\mathbf{0}}, \boldsymbol{u}^{\mathbf{1}}\right)>=\int_{0}^{T}\left[b^{\varepsilon}(\boldsymbol{u}, \boldsymbol{u})\right]_{0}^{1} d t
$$

and is an isomorphism. Thus, for any $\left(\boldsymbol{y}^{\mathbf{0}}, \boldsymbol{y}^{\mathbf{1}}\right)$ such that $\left(-\boldsymbol{y}^{\mathbf{1}}, \boldsymbol{y}^{\mathbf{0}}\right) \in \boldsymbol{V}^{\prime} \times \boldsymbol{H}$, there exists a control function $\boldsymbol{v}=\left(u_{1,1} \nu, u_{3,11}\right)$ on $\Sigma_{T}$ and the solution $\boldsymbol{y}$ of (13) is $\boldsymbol{y}=\boldsymbol{z}$ and satisfies $E(T, \boldsymbol{y})=0$. Finally, the hidden regularity implies that $b^{\varepsilon}(\boldsymbol{\varphi}, \boldsymbol{\varphi}) \in L^{1}\left(\Sigma_{T}\right)$. Therefore $v_{1}^{2}+\varepsilon^{2}\left(v_{3,1}-2 r^{-1} v_{1}\right)^{2}$ belongs to $L^{1}\left(\Sigma_{T}\right)$ and so $v_{1}, v_{3} \in L^{2}\left(\Sigma_{T}\right)$.

Remark 3 From the characterization (16), we obtain that the control $\boldsymbol{v}=\left(v_{1}, v_{3}\right)$ obtained from Theorem 4.1 is among all the admissible controls, the one which minimizes $\| v_{1}^{2}+$ $\varepsilon^{2}\left(v_{3,1}-2 r^{-1} v_{1}\right) / 3 \|_{L^{1}\left(\Sigma_{T}\right)}$.

As a summary, we obtain the exact null controllability of the arch by acting, on the extremities, on the tangential component $y_{1}$ through a Dirichlet control $v_{1}$ and on the normal component $y_{3}$ through a Neumann control $v_{3}$. In the next section, we address the numerical resolution of this control problem and highlight the influence of the parameter $\varepsilon$ and $r^{-1}$.

\section{Numerical experiments}

According to Section 4, the controllability problem is reduced, via the duality HUM method, to the solution of the following linear problem: find $\left(\boldsymbol{u}^{\mathbf{0}}, \boldsymbol{u}^{\mathbf{1}}\right) \in \boldsymbol{V} \times \boldsymbol{H}$ such that

$$
<\Lambda^{\varepsilon}\left(\boldsymbol{u}^{\mathbf{0}}, \boldsymbol{u}^{\mathbf{1}}\right),\left(\hat{\boldsymbol{u}}^{\mathbf{0}}, \hat{\boldsymbol{u}}^{\mathbf{1}}\right)>=<\left(\boldsymbol{y}^{\mathbf{1}},-\boldsymbol{y}^{\mathbf{0}}\right),\left(\hat{\boldsymbol{u}}^{\mathbf{0}}, \hat{\boldsymbol{u}}^{\mathbf{1}}\right)>_{\boldsymbol{V}^{\prime} \times \boldsymbol{H}, \boldsymbol{V} \times \boldsymbol{H}}, \quad \forall\left(\hat{\boldsymbol{u}}^{\mathbf{0}}, \hat{\boldsymbol{u}}^{\mathbf{1}}\right) \in \boldsymbol{V} \times \boldsymbol{H}
$$


which is equivalent to minimize the following functional $\mathcal{J}: \boldsymbol{V} \times \boldsymbol{H} \rightarrow \mathbb{R}$

$$
\begin{aligned}
\mathcal{J}^{\varepsilon}\left(\boldsymbol{u}^{\mathbf{0}}, \boldsymbol{u}^{\mathbf{1}}\right) & =\frac{1}{2}<\Lambda^{\varepsilon}\left(\boldsymbol{u}^{\mathbf{0}}, \boldsymbol{u}^{\mathbf{1}}\right),\left(\boldsymbol{u}^{\mathbf{0}}, \boldsymbol{u}^{\mathbf{1}}\right)>-<\left(\boldsymbol{y}^{\mathbf{1}},-\boldsymbol{y}^{\mathbf{0}}\right),\left(\boldsymbol{u}^{\mathbf{0}}, \boldsymbol{u}^{\mathbf{1}}\right)>_{V^{\prime} \times \boldsymbol{H}, \boldsymbol{V} \times \boldsymbol{H}} \\
& =\frac{1}{2} \int_{0}^{T}\left[b^{\varepsilon}(\boldsymbol{u}, \boldsymbol{u})\right]_{0}^{1} d t+\int_{\Omega} \boldsymbol{y}^{\mathbf{0}} \cdot \boldsymbol{u}^{\mathbf{1}} d \xi-<\boldsymbol{y}^{\mathbf{1}}, \boldsymbol{u}^{\mathbf{0}}>_{\boldsymbol{V}^{\prime}, \boldsymbol{V}} \\
& =\frac{1}{2} \int_{0}^{T}\left[u_{1,1}^{2}+\frac{\varepsilon^{2}}{3}\left(u_{3,11}-2 r^{-1} u_{1,1}\right)^{2}\right]_{0}^{1} d t+\int_{\Omega} \boldsymbol{y}^{\mathbf{0}} \cdot \boldsymbol{u}^{\mathbf{1}} d \xi-<\boldsymbol{y}^{\mathbf{1}}, \boldsymbol{u}^{\mathbf{0}}>_{V^{\prime}, \boldsymbol{V}} .
\end{aligned}
$$

Actually, $\mathcal{J}^{\varepsilon}$ is the conjugate function of $J^{\varepsilon}:\left(L^{2}\left(\Sigma_{T}\right)\right)^{2} \rightarrow \mathbb{R}$ defined by $J^{\varepsilon}(\boldsymbol{v})=1 / 2 \| v_{1}^{2}+$ $\varepsilon^{2}\left(v_{3,1}-2 r^{-1} v_{1}\right) / 3 \|_{L^{1}\left(\Sigma_{T}\right)}$ (see Remark 3).

Since $\mathcal{J}^{\varepsilon}$ is continuous, convexe and $(\boldsymbol{V} \times \boldsymbol{H})$-elliptic for $T$ and $r$ sufficiently large, the minimization can be done by a conjugate gradient (CG) algorithm (we refer to [13] for the wave equation).

In this section, we present some numerical experiments of the minimization of $\mathcal{J}^{\varepsilon}$ and highlight the influence of the curvature $r^{-1}$ and above all of the thickness $\varepsilon$. For the wave equation (we refer to $[3,13,21]$ and the references therein), the numerical approximation of the exact control is very sensitive to the choice of the approximation method and to the regularity of the initial data $\left(\boldsymbol{y}^{\mathbf{0}}, \boldsymbol{y}^{\mathbf{1}}\right)$. This sensitivity is likely to remain for our $2 \times 2$ system (1), at least for the Dirichlet control $v_{1}$ which formally converges to the control of the wave equation on $y_{1}$ when $r^{-1}$ vanishes (see Remark 2). Moreover, very little seems to be known on the quality of approximation of the Neumann control.

Consequently, in order to expect a convergent approximation, we consider only regular data involving first frequency components. Secondly, we regularize the control at time $t=0$ and $t=T$ : we introduce a compact support function $\rho \in C_{c}^{1}([0, T])$ - typically $\rho(t)=$ $\sin ^{2}(t \pi / T)$ - and search for a control under the form $\left(\rho v_{1}, \rho v_{3}\right)$. These two assumptions, meaningful from a mechanical viewpoint, allow to obtain convergent and robust numerical approximations (see Section 5.1). In this respect, since the weak adjoint solution $\boldsymbol{u}$ belongs to $C\left(0, T ; H_{0}^{1}(\omega) \times H_{0}^{2}(\omega)\right)$, a conformal $C^{0}-C^{1}$ finite element method is used: introducing a uniform subdivision $\left\{x_{i}\right\}_{(i=1, . ., J)}$ of $\omega=(0,1)$, we approximate $\boldsymbol{V}$ by the finite dimensional space

$\boldsymbol{V}_{\boldsymbol{h}}=\left\{\left(v_{1 h}, v_{3 h}\right) \in C^{0}(\bar{\omega}) \times C^{1}(\bar{\omega}),\left(v_{1 h}\right)_{\mid\left[x_{i}, x_{i+1}\right]} \in \mathbb{P}_{1},\left(v_{3 h}\right)_{\mid\left[x_{i}, x_{i+1}\right]} \in \mathbb{P}_{3}, \forall i=1, \ldots, J-1\right\}$

where $\mathbb{P}_{k}$ designates the space of the polynomials of degree $\leq k$. The time approximation is performed in a standard way using centered finite differences : in particular, we use a Newmark method to get an unconditionally stable scheme. The introduction of a $C^{1}$-finite element as well as the presence of a Neumann control is a major difference and new ingredient with respect to the numerical literature mainly devoted to the wave equation with Dirichlet boundary condition.

Two stopping criteria for the CG algorithm may be used: the first one is related to the residual $r^{(k)}$ at the iteration $k$, namely $r^{(k)} \leq \eta r^{(0)}, \eta>0$, permits to analyze the quality of the numerical approximation. The second one is related to the numerical energy $E_{h}$ of the 
system, namely $E_{h}(T) \leq \eta E_{h}(0)$, permits to analyze the efficiency of the control.

Remark 4 A key point in the conjugate gradient algorithm is the choice of the scalar product on $\boldsymbol{V} \times \boldsymbol{H}$ : in this respect, we have replaced the usual scalar product

$$
\left(\left(\boldsymbol{v}^{\mathbf{0}}, \boldsymbol{v}^{\mathbf{1}}\right),\left(\tilde{\boldsymbol{v}}^{\mathbf{0}}, \tilde{\boldsymbol{v}}^{\mathbf{1}}\right)\right)_{\boldsymbol{V} \times \boldsymbol{H}}=\int_{\omega}\left(v_{1,1}^{0} \tilde{v}_{1,1}^{0}+v_{3,11}^{0} \tilde{v}_{3,11}^{0}\right) d \xi+\int_{\omega}\left(v_{1}^{1} \tilde{v}_{1}^{1}+v_{3}^{1} \tilde{v}_{3}^{1}\right) d \xi
$$

by the more natural one :

$$
\left(\left(\boldsymbol{v}^{\mathbf{0}}, \boldsymbol{v}^{\mathbf{1}}\right),\left(\tilde{\boldsymbol{v}}^{\mathbf{0}}, \tilde{\boldsymbol{v}}^{\mathbf{1}}\right)\right)_{\boldsymbol{V} \times \boldsymbol{H}}=\int_{\omega} b^{\varepsilon}\left(\boldsymbol{v}^{\mathbf{0}}, \tilde{\boldsymbol{v}}^{\mathbf{0}}\right) d \xi+\int_{\omega}\left(v_{1}^{1} \tilde{v}_{1}^{1}+v_{3}^{1} \tilde{v}_{3}^{1}\right) d \xi
$$

Remark 5 One may improve the robustness of the algorithm (in particular reduce the number of iterations) by coupling the $C^{0}-C^{1}$ finite element scheme with a Bi-grid method: introduced in [13] for the wave equation, it consists in computing at each iteration the descent direction on a coarse mesh. We have observed that the Bi-grid method remains efficient in our context provided a careful extension (from the coarse to the fine mesh) of the nodal $C^{1}$ finite element approximation of $y_{3}$. The gain of robustness is very valuable when we are at the theoretical limit of controllability (see Section 5.3).

\subsection{Analysis with respect to $h$}

Before highlighting the influence of the parameters $\varepsilon$ and $r^{-1}$ on the controllability, it is essential to analyze our numerical approximation with respect to the discretization parameter $h$ and $d t$.

Let us first consider the following data : $\varepsilon=10^{-1}, r^{-1}=10^{-1}, T=3$. We assume that the control is acting on the left extremity $\xi=0$ of $\omega=(0,1)$ and we note $\Sigma_{T}^{0}=\{0\} \times(0, T)$. On the right extremity $\xi=1$, the arch is clamped. We start with the following simple initial condition (in the local curvilinear frame) $\left(\boldsymbol{y}^{\mathbf{0}}, \boldsymbol{y}^{\mathbf{1}}\right) \in \boldsymbol{H} \times \boldsymbol{V}^{\prime}$ :

$$
\boldsymbol{y}^{\mathbf{0}}=\left(y_{1}^{0}, y_{3}^{0}\right)=\left(\sin (\pi \xi), \sin ^{2}(\pi \xi)\right) ; \quad \boldsymbol{y}^{\mathbf{1}}=\left(y_{1}^{1}, y_{3}^{1}\right)=(0,0), \quad \xi \in \omega=(0,1) .
$$

We use the stopping criterion related to the residual $r^{(k)}$ with $\eta=10^{-8}$ and take $h=1 / 64$ and $\Delta t=h / 2$. Figure 1 depicts the initial condition of the system (8), minimum in $\boldsymbol{V} \times \boldsymbol{H}$ of the associated functional $\mathcal{J}$ (see 17). Figure 2-left represents the evolution with respect to time of the associated control $\boldsymbol{v}=\left(v_{1}, v_{3}\right) \in\left(L^{2}\left(\Sigma_{T}^{0}\right)\right)^{2}$. As explained previously, the control and its first derivative are zero at time $t=0$ and $t=T$. Figure 2-right represents the evolution of the energy (non monotonous). We check the (numerical) controllability by computing the ratio $E_{h}(T) / E_{h}(0) \approx 4.75 \times 10^{-5}$. Figure 3 represents the evolution of the controlled tangential $y_{1}$ and normal $y_{3}$ displacement on $Q_{T}$ (attached to the local basis). Finally, for $r^{-1}=1 / 5$ and $r^{-1}=1 / 10$, Figure 4 represents the evolution of the circular arch submitted to the initial position $\boldsymbol{y}^{\mathbf{0}}(\boldsymbol{x})=y_{1}^{0} \boldsymbol{\tau}(\xi)+y_{3}^{0} \boldsymbol{\nu}(\xi)$ and to the initial velocity $\boldsymbol{y}^{\mathbf{1}}(\boldsymbol{x})=y_{1}^{1} \boldsymbol{\tau}(\xi)+y_{3}^{1} \boldsymbol{\nu}(\xi)$.

We then collect in Table 1 the evolution of several relevant quantities with respect to $h=$ 

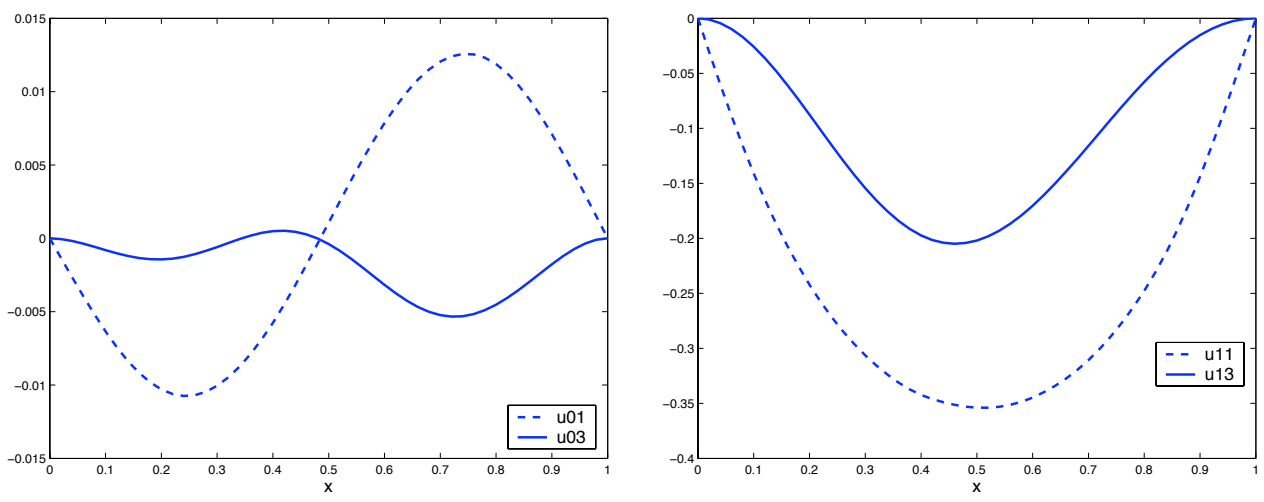

Figure 1: $T=3$ - Initial conditions $\boldsymbol{u}^{\mathbf{0}}=\left(u_{1}^{0}, u_{3}^{0}\right)$ (Left) and $\boldsymbol{u}^{\mathbf{1}}=\left(u_{1}^{1}, u_{3}^{1}\right)$ (Right) of the system (8) and minimum of the functional $\mathcal{J}$ (defined by eq. 17 ).
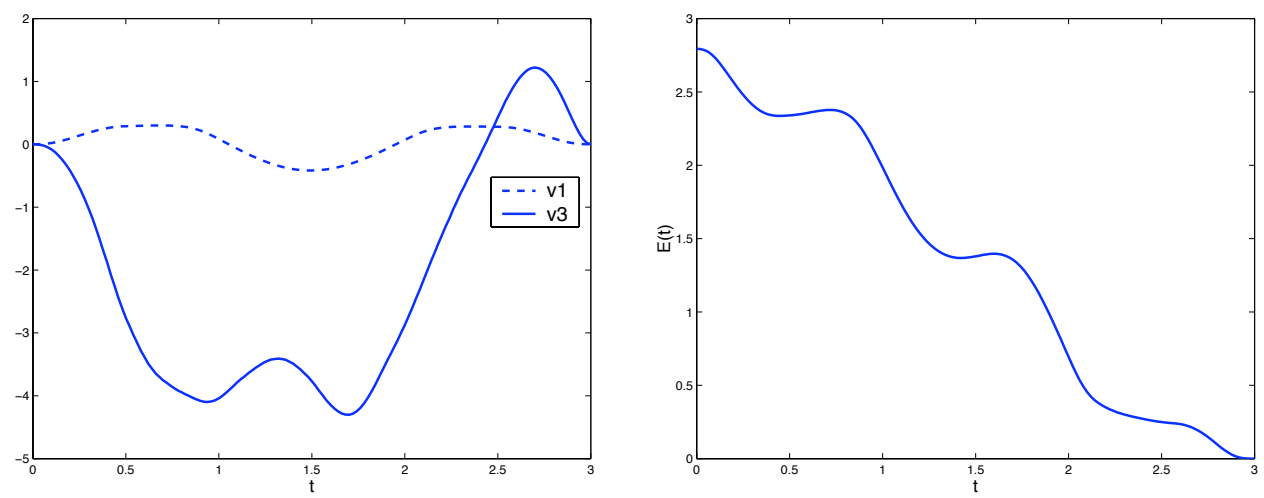

Figure 2: $T=3$ - Left - Compact support control $\left(v_{1}, v_{3}\right)=\left(\partial_{\nu} u_{1}, \partial_{\nu \nu}^{2} u_{3}\right)$ on $\Sigma_{T}^{0}$; Right Energy $E_{h}(t, \boldsymbol{y})$ vs $t$.
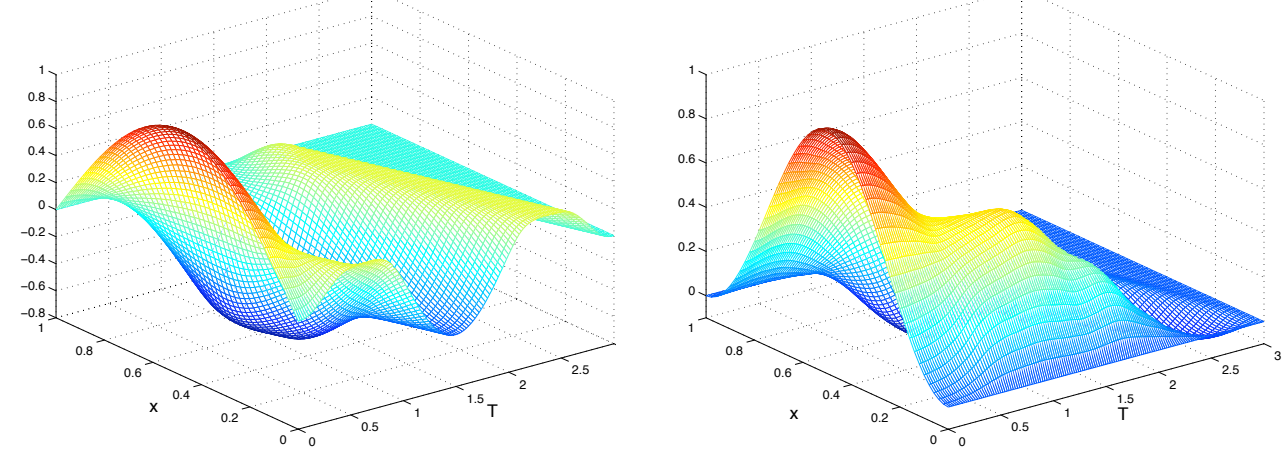

Figure 3: $T=3$ - Controlled displacement $y_{1}$ (Left) and $y_{3}$ (Right) on $Q_{T}$. 

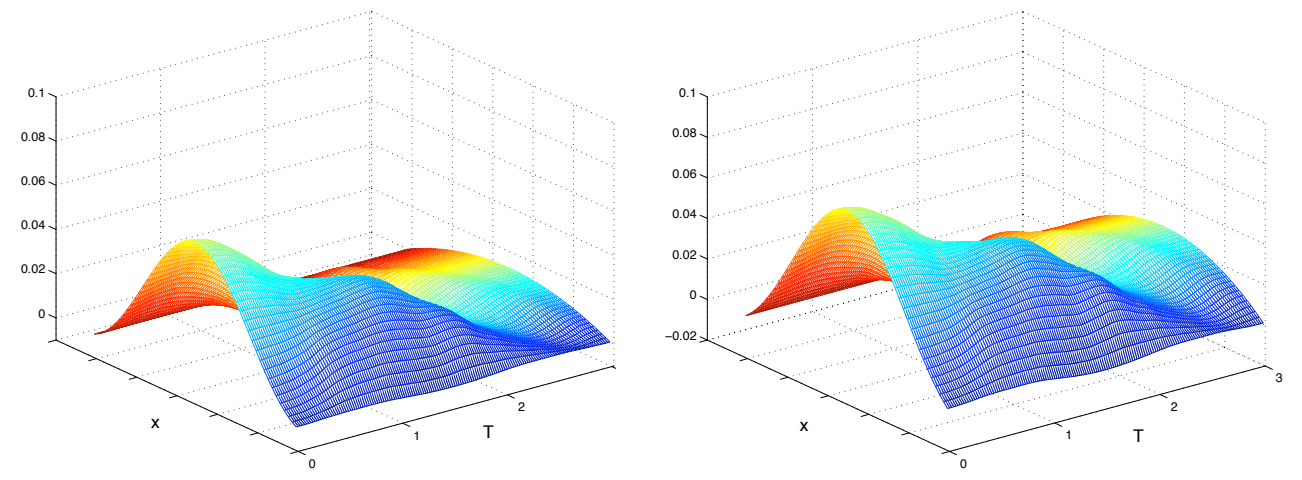

Figure 4: $T=3$ - Evolution of the controlled cylindrical arch vs $t \in[0,3]$ for $r^{-1}=1 / 10$ (Left) and $r^{-1}=1 / 5$ (Right).

$2^{-k}, k=4, \ldots, 7$. In particular, the quantities $\left\|y_{1}^{0}-y_{1 h}^{0}\right\|_{L^{2}(\omega)} /\left\|y_{1}^{0}\right\|_{L^{2}(\omega)}$ and $\left\|y_{1}^{1}-y_{1 h}^{1}\right\|_{H^{-1}(\omega)}$ measure how well the initial conditions $\left(\boldsymbol{y}^{\mathbf{0}}, \boldsymbol{y}^{\mathbf{1}}\right)$ are recovered by the algorithm (recall that they are obtained from the system (15)). The $H^{-1}$ norm of any scalar function $v$ is computed writting that $\|v\|_{H^{-1}(\omega)}=|w|_{H^{1}(\omega)}$ with $|w|_{H^{1}(\omega)}=\left(\int_{\omega}|\nabla w|^{2} d \xi\right)^{1 / 2}$ and $w \in H_{0}^{1}(\omega)$ is the solution of the Dirichlet problem : $-\Delta w=v$ in $\omega, w=0$ on $\partial \omega$. Similarly, the $H^{-2}$ norm of any scalar function $v$ is computed writing that $\|v\|_{H^{-2}(\omega)}=|w|_{H^{2}(\omega)}$ with $|w|_{H^{2}(\omega)}=\left(\int_{\omega}|\Delta w|^{2} d \xi\right)^{1 / 2}$ and $w \in H_{0}^{2}(\omega)$ is the solution of the problem : $\Delta^{2} w=v$ in $\omega$, $w=\partial_{\nu} w=0$ on $\partial \omega$.

The evolution of these quantities suggest the convergence of the approximation as $h$ goes to zero. Moreover, we observe that the number of iterations needed to reach the convergence (the criterion $r^{(k)} \leq 10^{-8} r^{(0)}$ ) is independent of the mesh size $h$. We may also compute the order of convergence of the controls (taking as reference solution the solution obtained with the small mesh size $h=2^{-8}$ ): we obtain the following order

$$
\begin{aligned}
& \left\|u_{1}-u_{1 h}\right\|_{L^{2}(\Omega)}=O\left(h^{2.07}\right), \quad\left|u_{1}-u_{1 h}\right|_{H^{1}(\Omega)}=O\left(h^{0.97}\right), \\
& \left\|u_{3}-u_{3 h}\right\|_{L^{2}(\Omega)}=O\left(h^{2.12}\right), \quad\left|u_{3}-u_{3 h}\right|_{H^{2}(\Omega)}=O\left(h^{1.07}\right), \\
& \left\|v_{1}-v_{1 h}\right\|_{L^{2}\left(\Sigma_{T}^{0}\right)}=O\left(h^{0.98}\right), \quad\left\|v_{3}-v_{3 h}\right\|_{L^{2}\left(\Sigma_{T}^{0}\right)}=O\left(h^{1.01}\right)
\end{aligned}
$$

in full agreement with the order of the scheme used on a uniform discretization.

Remark 6 Following [21] for the wave equation, one may exhibit the loss of convergence of the approximated controls by considering less regular initial data (we also refer to [13]).

Being confident about the quality of the numerical approximation when regular initial data are involved, we now study the influence of the parameters on the uniform controllability result. 


\begin{tabular}{|c|c|c|c|c|}
\hline$h$ & $1 / 16$ & $1 / 32$ & $1 / 64$ & $1 / 128$ \\
\hline \# CG iterations & 12 & 9 & 9 & 9 \\
\hline$\frac{\left\|y_{1}^{0}-y_{1 h}^{0}\right\|_{L^{2}(\Omega)}}{\left\|y_{1}^{0}\right\|_{L^{2}(\Omega)}}$ & $3.21 \times 10^{-3}$ & $6.28 \times 10^{-4}$ & $1.11 \times 10^{-4}$ & $3.40 \times 10^{-5}$ \\
\hline$\left\|y_{1}^{1}-y_{1 h}^{1}\right\|_{H^{-1}(\Omega)}$ & $1.39 \times 10^{-2}$ & $3.78 \times 10^{-3}$ & $1.25 \times 10^{-3}$ & $3.87 \times 10^{-5}$ \\
\hline$\frac{\left\|y_{3}^{0}-y_{3 h}^{0}\right\|_{L^{2}(\Omega)}}{\left\|y_{3}^{0}\right\|_{L^{2}(\Omega)}}$ & $2.58 \times 10^{-4}$ & $5.70 \times 10^{-5}$ & $3.20 \times 10^{-5}$ & $3.08 \times 10^{-5}$ \\
\hline$\left\|y_{3}^{1}-y_{3 h}^{1}\right\|_{H^{-2}(\Omega)}$ & $7.88 \times 10^{-5}$ & $9.52 \times 10^{-6}$ & $7.99 \times 10^{-7}$ & $7.74 \times 10^{-7}$ \\
\hline$\left\|y_{1 h}(T)\right\|_{L^{2}(\Omega)}$ & $2.92 \times 10^{-3}$ & $5.28 \times 10^{-4}$ & $9.61 \times 10^{-5}$ & $2.64 \times 10^{-5}$ \\
\hline$\left\|y_{3 h}(T)\right\|_{L^{2}(\Omega)}$ & $1.93 \times 10^{-3}$ & $2.73 \times 10^{-4}$ & $8.52 \times 10^{-5}$ & $3.15 \times 10^{-5}$ \\
\hline$\left\|y_{1 h}^{\prime}(T)\right\|_{H^{-1}(\Omega)}$ & $5.20 \times 10^{-3}$ & $2.98 \times 10^{-3}$ & $7.88 \times 10^{-4}$ & $2.33 \times 10^{-4}$ \\
\hline$\left\|y_{3 h}^{\prime}(T)\right\|_{H^{-2}(\Omega)}$ & $4.73 \times 10^{-4}$ & $1.85 \times 10^{-4}$ & $1.11 \times 10^{-4}$ & $5.99 \times 10^{-5}$ \\
\hline$\left\|v_{1 h}\right\|_{L^{2}(\{0\} \times(0, T))}$ & $4.153 \times 10^{-1}$ & $4.177 \times 10^{-1}$ & $4.183 \times 10^{-1}$ & $4.184 \times 10^{-1}$ \\
\hline$\left\|v_{3 h}\right\|_{L^{2}(\{0\} \times(0, T))}$ & 4.847 & 4.846 & 4.845 & 4.845 \\
\hline$\left|u_{1 h}^{0}\right|_{H^{1}(\Omega)}$ & $4.83 \times 10^{-2}$ & $5.08 \times 10^{-2}$ & $5.14 \times 10^{-2}$ & $5.15 \times 10^{-2}$ \\
\hline$\left|u_{3 h}^{0}\right|_{H^{2}(\Omega)}$ & $1.902 \times 10^{-1}$ & $1.978 \times 10^{-1}$ & $1.993 \times 10^{-1}$ & $1.995 \times 10^{-1}$ \\
\hline$E_{h}(T) / E_{h}(0)$ & $3.78 \times 10^{-3}$ & $4.04 \times 10^{-4}$ & $4.75 \times 10^{-5}$ & $7.56 \times 10^{-6}$ \\
\hline
\end{tabular}

Table 1: Numerical approximations vs. $h=1 / 16,1 / 32,1 / 64$ and $h=1 / 128-\Delta t=h / 2$.

\subsection{Influence of the curvature $r^{-1}$ on the controllability}

The controllability result from Theorem 4.1 requires that the curvature - coupling parameter between $y_{1}$ and $y_{3}$ - be small enough $\left(r^{-1} \leq 1 / D(\varepsilon)\right)$. We highlight that this condition so called shallowness assumption - is also prescribed in the literature, both in the context of controllability and stabilisation $([4,11,14,20])$. Table 2 gives the norm of the control with respect to the curvature, from an almost rectilinear arch $\left(r^{-1}=\pi / 256\right)$ to a semicircle $\left(r^{-1}=\pi\right)$. We take $T=3, \varepsilon=1 / 10$, the initial condition (18) and assume that the control is active on the two extremities $\xi=0$ and $\xi=1$ of the arch. We observe that these norm increase with $r^{-1}$ : however, the controllability still holds even in the very curved situation so that the condition $r^{-1} \leq 1 / D(\varepsilon)$ of Theorem 4.1 is not restrictive in practice (remark that $\varepsilon=10^{-1}$ corresponds to a rather thick arch). The amount of work $\left\|b^{\varepsilon}(\boldsymbol{u}, \boldsymbol{u})\right\|_{L^{1}\left(\Sigma_{T}\right)}=\left\|v_{1}^{2}+\varepsilon^{2} / 3\left(v_{3}-2 r^{-1} v_{1}\right)^{2}\right\|_{L^{1}\left(\Sigma_{T}\right)}$ produced by the control increases smoothly with the curvature. Actually, spectral computation for the hemi-sphere performed in ([10]) (by symmetry, the analysis is reduced to a one-dimensional analysis) that the time $T=T(r)$ of controllability is of order of $r^{-1}$. The same result is obtained in [1] in the case $\varepsilon=0$. We also remark that the control $v_{1}$ acting on the tangential displacement is more active for large curvature. Finally, we also check that, when the curvature goes to zero, the two controls converge toward the control of the wave equation and beam equation (see Remark 2). 


\begin{tabular}{c|ccccc}
\hline$r^{-1}$ & $\pi / 256$ & $\pi / 64$ & $\pi / 16$ & $\pi / 4$ & $\pi$ \\
\hline \# CG iterations & 8 & 8 & 8 & 10 & 12 \\
$\left\|v_{1 h}\right\|_{L^{2}\left(\Sigma_{T}\right)}$ & $2.95 \times 10^{-1}$ & $2.94 \times 10^{-1}$ & $2.91 \times 10^{-1}$ & $2.51 \times 10^{-1}$ & $3.67 \times 10^{-1}$ \\
$\left\|v_{3 h}\right\|_{L^{2}\left(\Sigma_{T}\right)}$ & 3.372 & 3.376 & 3.439 & 4.175 & 3.033 \\
$\left\|b^{\varepsilon}(\boldsymbol{u}, \boldsymbol{u})\right\|_{L^{1}\left(\Sigma_{T}\right)}$ & $1.24 \times 10^{-1}$ & $1.24 \times 10^{-1}$ & $1.25 \times 10^{-1}$ & $1.29 \times 10^{-1}$ & $1.54 \times 10^{-1}$ \\
$E_{h}(T) / E_{h}(0)$ & $6.92 \times 10^{-6}$ & $7.01 \times 10^{-6}$ & $8.58 \times 10^{-6}$ & $5.07 \times 10^{-5}$ & $4.54 \times 10^{-5}$ \\
\hline
\end{tabular}

Table 2: Approximation of the control vs. the curvature $-\Sigma_{T}=\partial \omega \times(0, T)$.

\subsection{Loss of uniform controllability with respect to the thickness $\varepsilon^{-1}$}

As announced in the introduction, the controllability issue is more sensitive and subtle with respect to the thickness. We first highlight that the system (1) where the elasticity operator defined on $\omega \times-] \varepsilon / 2, \varepsilon / 2\left[\right.$ is replaced by the operator $\boldsymbol{A}^{\varepsilon}=\boldsymbol{A}_{\boldsymbol{M}}+\varepsilon^{2} / 3 \boldsymbol{A}_{\boldsymbol{F}}$ on the mid surface $\omega$ is an approximation of Koiter type assuming the variable $\varepsilon$ small enough (see $[6,23])$. It it therefore interesting to known the behavior of the control when $\varepsilon$ is arbitrarily small. We take the data of the previous section. We use now the stopping criterion on the energy, i.e. $E_{h}(T) \leq \eta E_{h}(0)$ - with $\eta=10^{-8}$, which permits to compare controls leading to a same level of (numerical) controllability. Figure 5 depicts the evolution of the $L^{2}$-norm of the controls $v_{1}$ and $v_{3}$ with respect to $\varepsilon$ for $T=3,4$ and $T=5$. Table 3 gives some numerical values. It appears clearly that these norm blow up as $\varepsilon$ goes to zero: precisely, as soon as $\varepsilon$ is small enough, the controllability properties does not hold anymore: for $T=3,4$ and $T=5$, the critical thickness is approximatively $\varepsilon=0.037,0.048$ and 0.058 respectively so that the minimal time of controllability - as a function of $\varepsilon$ - behaves like $T(\varepsilon) \approx O\left(\varepsilon^{-1}\right)$. This illustrates the lack of uniform controllability of the system (1) with respect to $\varepsilon$ and is in full agreement with [11]. This also validates somehow our numerical approximation. Remark that the non boundeness of the control observed in Table 3 is not in contradiction with Theorem 4.1 because $T$ is here fixed independent of $\varepsilon$. When $T$ behaves like $O\left(\varepsilon^{-1}\right)$, $\left\|v_{1}\right\|_{L^{2}\left(\Sigma_{T}\right)}$ and $\left\|\varepsilon v_{3}\right\|_{L^{2}\left(\Sigma_{T}\right)}$ remain bounded (see Table 4). Since $\varepsilon$ varies here, it is more relevant to consider in Table 4 the quantity $\varepsilon v_{3}$ (instead of $v_{3}$ ) which appears in the bilinear form $b^{\varepsilon}$.

\begin{tabular}{c|cccc}
\hline & $\varepsilon=1 / 10$ & $\varepsilon=1 / 20$ & $\varepsilon=1 / 30$ & $\varepsilon=1 / 40$ \\
\hline \# CG iterations & 8 & 9 & 16 & n.c. \\
$\left\|v_{1 h}\right\|_{L^{2}\left(\Sigma_{T}\right)}$ & $2.942 \times 10^{-1}$ & $2.952 \times 10^{-1}$ & $3.095 \times 10^{-1}$ & n.c. \\
$\left\|\varepsilon v_{3 h}\right\|_{L^{2}\left(\Sigma_{T}\right)}$ & $3.38 \times 10^{-1}$ & $3.56 \times 10^{-1}$ & $8.52 \times 10^{-1}$ & n.c. \\
$\left\|b^{\varepsilon}(\boldsymbol{u}, \boldsymbol{u})\right\|_{L^{1}\left(\Sigma_{T}\right)}$ & $1.24 \times 10^{-1}$ & $1.295 \times 10^{-1}$ & $3.37 \times 10^{-1}$ & n.c. \\
$E_{h}(T) / E_{h}(0)$ & $7.29 \times 10^{-6}$ & $3.36 \times 10^{-5}$ & $1.90 \times 10^{-3}$ & n.c. \\
\hline
\end{tabular}

Table 3: Approximation of the control vs. $\varepsilon-T=3-C=\pi / 32$ - n.c. stands for non controllability.

The loss of controllability as $\varepsilon$ goes to zero is related to the property of the operator $\boldsymbol{A}_{\boldsymbol{M}}$ 

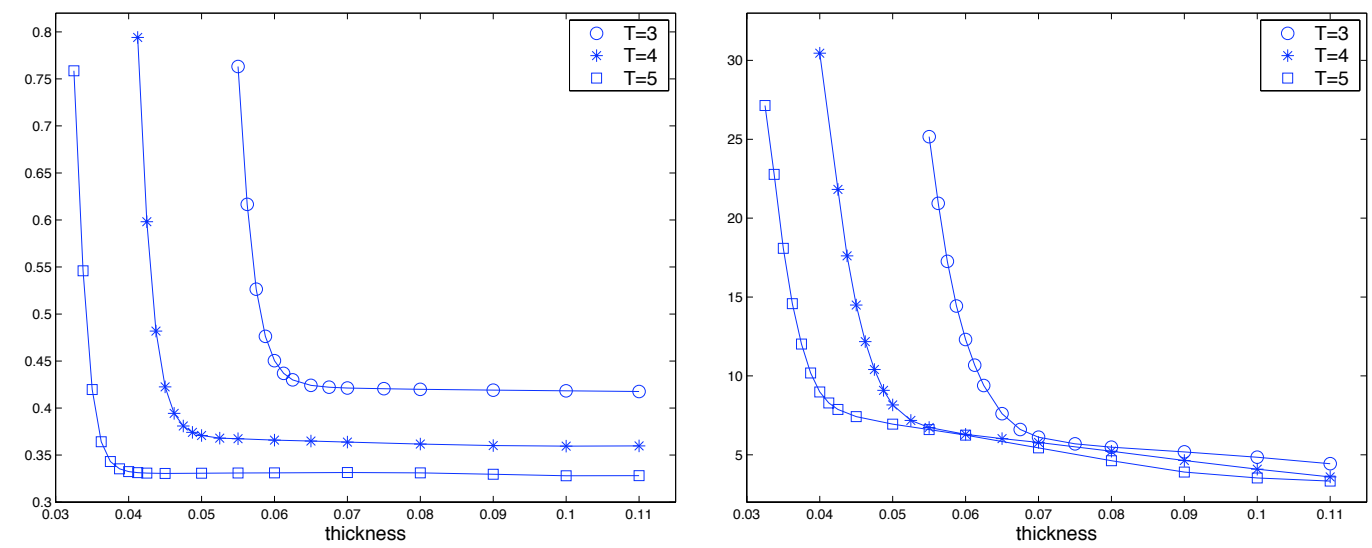

Figure 5: $L^{2}$-norm $\left\|v_{1}\right\|_{L^{2}\left(\Sigma_{T}\right)}\left(\right.$ Left) and $\left\|v_{3}\right\|_{L^{2}\left(\Sigma_{T}\right)}$ (Right) vs. the thickness $\varepsilon$.

\begin{tabular}{c|cccc}
\hline & $\varepsilon=1 / 10$ & $\varepsilon=1 / 20$ & $\varepsilon=1 / 30$ & $\varepsilon=1 / 40$ \\
\hline \# CG iterations & 8 & 8 & 8 & 8 \\
$\left\|v_{1 h}\right\|_{L^{2}\left(\Sigma_{T}\right)}$ & $2.942 \times 10^{-1}$ & $2.551 \times 10^{-1}$ & $2.302 \times 10^{-1}$ & $2.151 \times 10^{-1}$ \\
$\left\|\varepsilon v_{3 h}\right\|_{L^{2}\left(\Sigma_{T}\right)}$ & $3.38 \times 10^{-1}$ & $2.49 \times 10^{-1}$ & $2.41 \times 10^{-1}$ & $2.49 \times 10^{-1}$ \\
$\left\|b^{\varepsilon}(\boldsymbol{u}, \boldsymbol{u})\right\|_{L^{1}\left(\Sigma_{T}\right)}$ & $1.24 \times 10^{-1}$ & $8.57 \times 10^{-2}$ & $7.20 \times 10^{-2}$ & $6.70 \times 10^{-2}$ \\
$E_{h}(T) / E_{h}(0)$ & $7.29 \times 10^{-6}$ & $1.04 \times 10^{-5}$ & $2.14 \times 10^{-4}$ & $1.38 \times 10^{-4}$ \\
\hline
\end{tabular}

Table 4: Approximation of the control vs. $\varepsilon-T(\varepsilon)=3 /(10 \varepsilon)-C=\pi / 32$.

which formally appears when $\varepsilon$ is zero. The system (1) then reduces to

$$
\left\{\begin{array}{lr}
\boldsymbol{y}^{\prime \prime}+\boldsymbol{A}_{\boldsymbol{M}} \boldsymbol{y}=\mathbf{0} & \text { in } Q_{T} \\
y_{1}=0 & \text { on } \Sigma_{T} \\
\left(\boldsymbol{y}(\cdot, 0), \boldsymbol{y}^{\prime}(\cdot, 0)\right)=\left(\boldsymbol{y}^{\mathbf{0}}, \boldsymbol{y}^{\mathbf{1}}\right) & \text { in } \omega
\end{array}\right.
$$

with any boundary condition on the variable $y_{3}$ (so that $v_{1}$ is the only control for the two components). We point out that when $\varepsilon$ goes to zero, the boundary conditions $\varepsilon y_{3}=$ $\varepsilon y_{3,1}=0$ on $\Sigma_{T}$ on the normal displacement degenerate, and $H^{2}(\omega)$ is replaced by $L^{2}(\omega)$ without trace on $\partial \omega$. The study performed in [1] (extended in [2] to a two-dimensional setting) reveals that this limit system is not uniformly controllable (with respect to the data $\left.\left(\boldsymbol{y}^{\mathbf{0}}, \boldsymbol{y}^{\mathbf{1}}\right)\right)$ : the kernel $\operatorname{Ker} \boldsymbol{A}_{\boldsymbol{M}}$ is of infinite dimension (0 belongs to the essential spectrum of the operator $\boldsymbol{A}_{\boldsymbol{M}}$ ). The controllability, through a Dirichlet boundary control on $y_{1}$ holds uniformly (only) for all data in the orthogonal of $\operatorname{Ker} \boldsymbol{A}_{\boldsymbol{M}}$ :

$$
\left(\operatorname{Ker} \boldsymbol{A}_{M}\right)^{\perp}=\left\{\left(\psi_{1}, \psi_{3}\right) \in H_{0}^{1}(\omega) \times L^{2}(\omega), r^{-1} \psi_{1}+\psi_{3,1}=0 \text { in } H^{-1}(\omega)\right\}
$$

When $\varepsilon$ goes to zero, the operator $\boldsymbol{A}^{\varepsilon}$ loses his compactness property so that the minimal controllability time $T(\varepsilon)$ blows up. According to the study of the limit case, one can wonder whether or not the controllability for (1) holds uniformly with respect to $\varepsilon$ if $\left(\boldsymbol{y}^{\mathbf{0}}, \boldsymbol{y}^{\mathbf{1}}\right)$ belongs 
to the non empty space $\operatorname{Ker} \boldsymbol{A}_{\boldsymbol{M}} \cap\left(\boldsymbol{H} \cap \boldsymbol{V}^{\prime}\right)$. Remark that the initial condition (18) does not enjoy this property because $r^{-1} y_{1}^{0}+y_{3,1}^{0}=\sin (\pi \xi)\left(r^{-1}+2 \pi \cos (\pi \xi)\right) \neq 0$. For instance, we may consider the following initial data in $\left(\operatorname{Ker} \boldsymbol{A}_{\boldsymbol{M}}\right)^{\perp} \cap\left(\boldsymbol{H} \times \boldsymbol{V}^{\prime}\right)$

$$
\boldsymbol{y}^{\mathbf{0}}=\left(y_{1}^{0}, y_{3}^{0}\right)=\left(-2 \pi \cos (\pi \xi) \sin (\pi \xi), r^{-1} \sin ^{2}(\pi \xi)\right) ; \quad \boldsymbol{y}^{\mathbf{1}}=\boldsymbol{y}^{\mathbf{0}}, \quad \xi \in \omega=(0,1) .
$$

Then, we minimize the following functional with respect to $\left(\boldsymbol{u}^{\mathbf{0}}, \boldsymbol{u}^{\mathbf{1}}\right)$ in $\boldsymbol{V} \times \boldsymbol{H}$ :

$$
\mathcal{J}_{\eta}\left(\boldsymbol{u}^{\mathbf{0}}, \boldsymbol{u}^{\mathbf{1}}\right)=\mathcal{J}\left(\boldsymbol{u}^{\mathbf{0}}, \boldsymbol{u}^{\mathbf{1}}\right)+\eta^{-1}\left\|r^{-1} u_{1}^{0}+u_{3,1}^{0}\right\|_{H_{0}^{1}(\omega)}^{2}+\eta^{-1}\left\|r^{-1} u_{1}^{1}+u_{3,1}^{1}\right\|_{H^{-1}(\omega)}^{2}
$$

where we add a penalized term in order to enforce (asymptotically with respect to $\varepsilon$ ) the initial condition $\left(\boldsymbol{u}^{\mathbf{0}}, \boldsymbol{u}^{\mathbf{1}}\right)$ to be in $\left(\operatorname{Ker} \boldsymbol{A}_{\boldsymbol{M}}\right)^{\perp}$ : in practice, we take $\eta=\varepsilon$. Table 5 collects the norm of the control in this case. We observe that the norm of $\varepsilon v_{3}$ is not bounded with respect to $\varepsilon$ and therefore, the control $\left(v_{1}, v_{3}\right)$ corresponding to that initial condition, does not converge toward the control of the longitudinal case (corresponding to $\varepsilon=0$, see [1]).

\begin{tabular}{c|cccc|c}
\hline & $\varepsilon=1 / 10$ & $\varepsilon=1 / 20$ & $\varepsilon=1 / 30$ & $\varepsilon=1 / 40$ & $" \varepsilon=0 "$ \\
\hline \# CG iterations & 5 & 8 & 14 & 75 & 11 \\
$\left\|v_{1 h}\right\|_{L^{2}\left(\Sigma_{T}\right)}$ & 1.121 & 1.124 & 1.127 & 1.128 & 1.251 \\
$\left\|\varepsilon v_{3 h}\right\|_{L^{2}\left(\Sigma_{T}\right)}$ & $9.99 \times 10^{-2}$ & $8.55 \times 10^{-2}$ & $2.14 \times 10^{-1}$ & $6.02 \times 10^{0}$ & 0. \\
$E_{h}(T) / E_{h}(0)$ & $5.23 \times 10^{-6}$ & $5.43 \times 10^{-6}$ & $3.05 \times 10^{-5}$ & $5.32 \times 10^{-5}$ & $4.85 \times 10^{-7}$ \\
\hline
\end{tabular}

Table 5: Approximation of the control vs. $\varepsilon-T=3-r^{-1}=\pi / 32$ with the initial condition (20) element of $\left(\operatorname{Ker} \boldsymbol{A}_{\boldsymbol{M}}\right)^{\perp} \cap\left(\boldsymbol{H} \times \boldsymbol{V}^{\prime}\right)$.

This suggest that it is not enough to eliminate the initial data in $\operatorname{Ker} \boldsymbol{A}_{\boldsymbol{M}}$ to obtain a uniform convergence with respect to the thickness of the arch. This phenomenon is due to the fact the spectrum $\sigma\left(\boldsymbol{A}^{\varepsilon}\right)$ of $\boldsymbol{A}^{\varepsilon}$ does not converge toward $\sigma\left(\boldsymbol{A}_{\boldsymbol{M}}\right)=\{0\} \cup\left\{r^{-2}+(k \pi)^{2}\right\}_{k \geq 0}$ as $\varepsilon \rightarrow 0$. This fact, explained in detailled in [23] is due to two things : i) the fact that the limit operator $\boldsymbol{A}_{\boldsymbol{M}}$ posseses an essential spectrum; ii) the fact that the boundary conditions on $y_{3}$ degenerate when $\varepsilon \rightarrow 0$. This implies a densification of the spectrum $\sigma\left(\boldsymbol{A}^{\varepsilon}\right)$ along $\mathbb{R}^{+}$: precisely (see [23], chapter 10 and the appendix)

$$
\lim _{\varepsilon \rightarrow 0} \overline{\sigma\left(\boldsymbol{A}^{\varepsilon}\right)}=\mathbb{R}^{+} \cup\{+\infty\} \neq \sigma\left(\boldsymbol{A}_{\boldsymbol{M}}\right) .
$$

Remark that this limit coincides precisely with the convex envelop of $\sigma_{\text {ess }}\left(\boldsymbol{A}_{\boldsymbol{M}}\right)=\{0,+\infty\}$. We also emphasize that the situation here is much complex than the situation considered in [10], where for symmetry property, there is no degeneracy of the boundary condition, and therefore no densification of the spectrum as $\varepsilon$ goes to zero. We refer to the Appendix where we characterize deeper the spectrum $\sigma\left(\boldsymbol{A}^{\varepsilon}\right)$. It appears the part $\sigma\left(\boldsymbol{A}^{\varepsilon}\right) \subset\left(r^{-2}, \infty\right)$ is composed of two sets $\sigma^{\epsilon,+}$ and $\sigma^{\epsilon,-}$ defined as follows :

$$
\begin{aligned}
& \sigma^{\epsilon,+}=\left\{\lambda_{k}^{\epsilon}>r^{-2}, \boldsymbol{A}^{\epsilon} \boldsymbol{\psi}_{\boldsymbol{k}}^{\epsilon,+}=\lambda_{k}^{\epsilon} \boldsymbol{\psi}_{\boldsymbol{k}}^{\epsilon,+}, \lambda_{k}^{\epsilon} \rightarrow r^{-2}+(k \pi)^{2}, \boldsymbol{\psi}_{\boldsymbol{k}}^{\boldsymbol{\epsilon},+} \rightarrow \boldsymbol{v}_{\boldsymbol{k}} \text { in } \boldsymbol{H}\right\}, \\
& \sigma^{\epsilon,-}=\left\{\lambda_{k}^{\epsilon}>r^{-2}, \boldsymbol{A}^{\epsilon} \boldsymbol{\psi}_{\boldsymbol{k}}^{\epsilon,-}=\lambda_{k}^{\epsilon} \boldsymbol{\psi}_{\boldsymbol{k}}^{\epsilon,-}, \lambda_{k}^{\epsilon} \rightarrow \mu_{k} \notin \sigma\left(\boldsymbol{A}_{\boldsymbol{M}}\right), \boldsymbol{\psi}_{\boldsymbol{k}}^{\epsilon,-} \rightarrow \mathbf{0} \text { in } \boldsymbol{H}\right\} .
\end{aligned}
$$


The first one is related to the spectrum of the limit operator $\boldsymbol{A}_{\boldsymbol{M}}$ while the second is composed of spurious elements. The second set densifies over $\left(r^{-2}, \infty\right)$ as $\epsilon \rightarrow 0$ (see Figure 7) and prevents from uniform spectral gap. Using Ingham type argument (see [2] in a similar context), one may show that controls $v^{\varepsilon}$ for system (1) are uniformly bounded and convergent toward control of (19) if and only if the intial data $\boldsymbol{y}^{\mathbf{0}}, \boldsymbol{y}^{\mathbf{1}}$ are generated by eigenfunctions associated with $\sigma^{\epsilon,+}$. This proof out of the scope of this work will be presented in a distinct work. Remark however that the approximate controllability problem, which consists to drive the state $\left(\boldsymbol{y}(\cdot, T), \boldsymbol{y}^{\prime}(\cdot, T)\right)$ in a neighborhood (independant of $\varepsilon$ ) of $(\mathbf{0}, \mathbf{0})$ holds uniformly w.r.t. $\varepsilon$ out of $\operatorname{Ker} \boldsymbol{A}_{\boldsymbol{M}}$. This is due to the fact that the energy $E\left(\boldsymbol{\psi}_{\boldsymbol{k}}^{\epsilon,-}, \boldsymbol{\psi}_{\boldsymbol{k}}^{\epsilon,-}\right)$ corresponding to the spurious modes $\boldsymbol{\psi}_{\boldsymbol{k}}^{\epsilon,-}$ converges to zero as $\varepsilon \rightarrow 0$ (related to the convergence of the spectral family of the operator $\boldsymbol{A}^{\varepsilon}$; see once again [23]).

\section{Final remarks}

This work is a contribution to the challenging thematic of null controllability of elastic arches and shells. The elastic cylindrical arch described by a Bernoulli type model, is exactly controllable by two boundary controls acting respectively on the tangential and normal displacement. The analysis highlights the influence and interplay of the curvature $r^{-1}$ and the thickness $\varepsilon$ on the controllability. When $\varepsilon$ is strictly positive and fixed, the operator $\boldsymbol{A}^{\varepsilon}$ enjoys compactness properties so that the arch is uniformly controllable after a time $T\left(r^{-1}\right)$ which increases linearly with the curvature $r^{-1}$. When the thickness goes to zero, the spectrum $\sigma\left(\boldsymbol{A}^{\varepsilon}\right)$ densifies all along $\mathbb{R}^{+}$, what implies non uniform gap properties with respect to the thickness. The uniform controllability is then lost in $\boldsymbol{H} \times \boldsymbol{V}^{\prime}$ (see also [12]). This phenonenon, related to the non empty essential spectrum of the mixed order operator $\boldsymbol{A}_{\boldsymbol{M}}$ and to the degeneracy of the initial condition, emphasizes how subtle the subspace (included in $\boldsymbol{H} \times \boldsymbol{V}^{\prime}$ ) of uniformly controllable data is. Finally, when $r^{-1}=0$ and $\varepsilon>0$, the two uncoupled components of the displacement are uniformly controllable. The loss of controllability in the thickness is therefore enhanced by the curvature. A general arch described by any map $\phi$ (except the trivial map $\phi(\xi)=(1,0)$ ) enjoys a similar phenomenon (and in particular the loss of controllability as $\varepsilon$ goes to zero). In the case of shells, the asymptotic characterization of the subspace for which the controllability holds seems open and challenging. We refer to [2] for the analysis of a cylinder for which the operator $\boldsymbol{A}_{\boldsymbol{M}}$ has a kernel reduced to zero but an essential spectrum of the type $\left[0, r^{-2}\right]$. Similarly, it would be interesting for which class of data, the system 1 is null controllable with only one control.

Our numerical experiments are in full agreement with these properties. Morever, we highlight that few works are concerned with exact controllability of system, a fortiori when Dirichlet and Neumann boundary control are involved. In this longitudinal asymptotic situation, there is no locking phenomenon to expect. However, according to [21], we may expect a loss of controllability with respect to the discretization parameter. In this respect, it is interesting to analyze the influence of the parabolic (resp. hyperbolic) terms of the system on the approximation of the Dirichlet control $v_{1}$ (resp. Neumann control $v_{3}$ ). 


\section{Appendix: On the spectrum of the operator $A^{\epsilon}$ vs. $\varepsilon$}

We analyze in this appendix the behavior of the spectrum $\sigma\left(\boldsymbol{A}^{\boldsymbol{\varepsilon}}\right)$ and then highlight why the controllability is not uniform with respect to the thickness $\varepsilon$, even out $\operatorname{Ker} \boldsymbol{A}_{\boldsymbol{M}}$. For simplicity, we perform the computation replacing the operator $\boldsymbol{A}_{\boldsymbol{F}}$ by the operator $\boldsymbol{A}_{\boldsymbol{F}}^{\star \star}$ defined in (5). This does not change the qualitative properties of $\sigma\left(\boldsymbol{A}^{\varepsilon}\right)$. We note $\epsilon=\varepsilon^{2} / 3$. The spectral problem is then the following one, for $\phi=\left(\phi_{1}, \phi_{3}\right)$ :

$$
\begin{cases}\boldsymbol{A}_{\boldsymbol{M}} \boldsymbol{\phi}+\epsilon \boldsymbol{A}_{\boldsymbol{F}} \boldsymbol{\phi}=\lambda^{\epsilon} \boldsymbol{\phi} & \text { in } \omega \\ \boldsymbol{\phi}=0, \phi_{3,1}=0 & \text { on } \partial \omega\end{cases}
$$

which reads as follows :

$$
\left\{\begin{array}{lr}
-\phi_{1,11}-r^{-1} \phi_{3,1}=\lambda^{\epsilon} \phi_{1} & \text { in } \omega \\
r^{-1} \phi_{1}+r^{-2} \phi_{3}+\epsilon \phi_{3,1111}=\lambda^{\epsilon} \phi_{3} & \text { in } \omega \\
\phi=0, \phi_{3,1}=0 & \text { on } \partial \omega
\end{array}\right.
$$

The operator $\boldsymbol{A}^{\epsilon}$ is positive and self-adjoint, so that $\lambda^{\epsilon}$ belong to $\mathbb{R}_{+}^{\star}$ for all $\epsilon>0$ : we have the relation

$$
\lambda^{\epsilon}=\frac{\int_{\omega}\left[\left(\phi_{1,1}+r^{-1} \phi_{3}\right)^{2}+\epsilon\left(\phi_{3,11}\right)^{2}\right] d \xi}{\int_{\omega}\left(\phi_{1}^{2}+\phi_{3}^{2}\right) d \xi}=\frac{\left\|b^{\varepsilon}(\phi, \phi)\right\|_{L^{1}(\omega)}}{\|\phi\|_{\boldsymbol{H}}^{2}}, \quad \boldsymbol{\phi}=\left(\phi_{1}, \phi_{3}\right) \neq(0,0) .
$$

Differentiating with respect to the variable $\xi$ in $(21)$, we may separate $\phi_{1}$ from $\phi_{3}$ to obtain that they both solve the following sixth order ordinary differential equation :

$$
\epsilon \phi_{i}^{(6)}+\lambda \epsilon \phi_{i}^{(4)}-\lambda \phi_{i}^{(2)}-\lambda\left(\lambda-r^{-2}\right) \phi_{i}=0, \quad i=1,3
$$

so that introducing the corresponding characteristic equation $\epsilon X^{6}+\lambda \epsilon X^{4}-\lambda X^{2}-\lambda(\lambda-$ $\left.r^{-2}-\alpha\right)=0$, we have to determine the roots of the third order polynomial

$$
p(m)=m^{3}+\lambda m^{2}-\frac{\lambda}{\epsilon} m-\frac{\lambda}{\epsilon}\left(\lambda-r^{-2}\right), \quad m=X^{2} .
$$

In the sequel, we are interested by the eigenvalues $\lambda^{\epsilon}$ greater than $r^{-2}$. This implies that $p(0)<0$ and $p^{\prime}(0)<0$. Therefore, there is one root $R_{3}^{\epsilon}(\lambda) \in(0, \infty)$. Moreover, by studying the polynomial $q(m)=p(m)-p(0)=m\left(m^{2}+\lambda m-\lambda / \varepsilon\right)$, we observe that if $\epsilon$ is small enough, the two others roots are reals and simple, $\left.R_{1}^{\epsilon}(\lambda), R_{2}^{\epsilon}(\lambda) \in\right]-\infty, 0[$ so that

$$
\frac{\lambda^{2}-\sqrt{\lambda^{2}+4 \lambda / \epsilon}}{2}<R_{1}^{\epsilon}(\lambda)<R_{2}^{\epsilon}(\lambda)<0<R_{3}^{\epsilon}(\lambda)<\frac{\lambda^{2}+\sqrt{\lambda^{2}+4 \lambda / \epsilon}}{2} .
$$

Therefore, noting $r_{1}(\lambda)=\sqrt{-R_{1}^{\epsilon}(\lambda)}, r_{2}(\lambda)=\sqrt{-R_{2}^{\epsilon}(\lambda)}$ and $r_{3}(\lambda)=\sqrt{R_{3}^{\epsilon}(\lambda)}, \phi_{1}$ and $\phi_{3}$ are generated by $\left\{\cos \left(r_{1}(\lambda) \xi\right), \sin \left(r_{1}(\lambda) \xi\right), \cos \left(r_{2}(\lambda) \xi\right), \sin \left(r_{2}(\lambda) \xi\right), e^{r_{3}(\lambda) \xi}, e^{-r_{3}(\lambda) \xi}\right\}$. There 
exist some constants $A_{i}, B_{i} \in \mathbb{R}, i=1, \ldots, 6$ so that

$$
\begin{aligned}
& \phi_{1}(\xi)=A_{1} \cos \left(r_{1}(\lambda) \xi\right)+A_{2} \sin \left(r_{1}(\lambda) \xi\right)+A_{3} \cos \left(r_{2}(\lambda) \xi\right)+A_{4} \sin \left(r_{2}(\lambda) \xi\right)+A_{5} e^{r_{3}(\lambda) \xi}+A_{6} e^{-r_{3}(\lambda) \xi} \\
& \phi_{3}(\xi)=B_{1} \cos \left(r_{1}(\lambda) \xi\right)+B_{2} \sin \left(r_{1}(\lambda) \xi\right)+B_{3} \cos \left(r_{2}(\lambda) \xi\right)+B_{4} \sin \left(r_{2}(\lambda) \xi\right)+B_{5} e^{r_{3}(\lambda) \xi}+B_{6} e^{-r_{3}(\lambda) \xi} .
\end{aligned}
$$

Reporting these expressions in $(21)$ and noting $\left(r_{i}(\lambda) \neq 0\right)$

$$
K_{1}=\frac{r_{1}(\lambda)^{2}-\lambda}{C r_{1}(\lambda)}, \quad K_{2}=\frac{r_{2}(\lambda)^{2}-\lambda}{C r_{2}(\lambda)}, \quad K_{3}=\frac{r_{3}(\lambda)^{2}+\lambda}{C r_{3}(\lambda)}
$$

we then get the relations $\left(B_{1}, B_{2}\right)=K_{1}\left(-A_{2}, A_{1}\right),\left(B_{3}, B_{4}\right)=K_{2}\left(-A_{4}, A_{3}\right)$ and $\left(B_{5}, B_{6}\right)=$ $K_{3}\left(-A_{5}, A_{6}\right)$ so that

$$
\begin{aligned}
\left(\begin{array}{l}
\phi_{1}(\xi) \\
\phi_{3}(\xi)
\end{array}\right)=A_{1} & \left(\begin{array}{r}
\cos \left(r_{1}(\lambda) \xi\right) \\
K_{1} \sin \left(r_{1}(\lambda) \xi\right)
\end{array}\right)+A_{2}\left(\begin{array}{r}
\sin \left(r_{1}(\lambda) \xi\right) \\
-K_{1} \cos \left(r_{1}(\lambda) \xi\right)
\end{array}\right)+A_{3}\left(\begin{array}{r}
\cos \left(r_{2}(\lambda) \xi\right) \\
K_{2} \sin \left(r_{2}(\lambda) \xi\right)
\end{array}\right) \\
& +A_{4}\left(\begin{array}{r}
\sin \left(r_{2}(\lambda) \xi\right) \\
-K_{2} \cos \left(r_{2}(\lambda) \xi\right)
\end{array}\right)+A_{5}\left(\begin{array}{r}
e^{\left.r_{3}(\lambda) \xi\right)} \\
-K_{3} e^{r_{3}(\lambda) \xi}
\end{array}\right)+A_{6}\left(\begin{array}{r}
e^{-r_{3}(\lambda) \xi} \\
K_{3} e^{-r_{3}(\lambda) \xi}
\end{array}\right)
\end{aligned}
$$

Finally, taking into account the boundary conditions, we get that $\lambda^{\epsilon} \geq r^{-2}$ solves the equation $\operatorname{det}\left(M\left(\lambda^{\epsilon}\right)\right)=0$ with (we note $r_{i}$ for $r_{i}(\lambda), i=1,2,3$ )

$$
M(\lambda)=\left(\begin{array}{cccccc}
1 & 0 & 1 & 0 & 1 & 1 \\
\cos \left(r_{1}\right) & \sin \left(r_{1}\right) & \cos \left(r_{2}\right) & \sin \left(r_{2}\right) & e^{r_{3}} & e^{-r_{3}} \\
0 & -K_{1} & 0 & -K_{2} & -K_{3} & K_{3} \\
K_{1} \sin \left(r_{1}\right) & -K_{1} \cos \left(r_{1}\right) & K_{2} \sin \left(r_{2}\right) & -K_{2} \cos \left(r_{2}\right) & -K_{3} e^{r_{3}} & K_{3} e^{-r_{3}} \\
K_{1} r_{1} & 0 & K_{2} r_{2} & 0 & -K_{3} r_{3} & -K_{3} r_{3} \\
K_{1} r_{1} \cos \left(r_{1}\right) & K_{1} r_{1} \sin \left(r_{1}\right) & K_{2} r_{2} \cos \left(r_{2}\right) & r_{2} K_{2} \sin \left(r_{2}\right) & -K_{3} r_{3} e^{r_{3}} & -K_{3} r_{3} e^{-r_{3}}
\end{array}\right) .
$$

Let us analyze the behavior of $r_{i}$ with respect to $\epsilon$. Using Cardan formulae, the roots $R_{1}, R_{2}, R_{3}$ of the third order polynomial $p$ defined in (22) takes the following expression

$$
R_{1}^{\epsilon}(\lambda), R_{2}^{\epsilon}(\lambda)=\frac{-\left(\lambda+R_{3}^{\epsilon}(\lambda)\right) \mp \sqrt{\Delta}}{2}, \quad R_{3}^{\epsilon}(\lambda)=2 \rho^{1 / 3} \cos \left(\frac{\theta}{3}\right)-\frac{\lambda}{3}
$$

with

$$
\left\{\begin{array}{l}
\rho=\left(-\frac{s}{3}\right)^{3 / 2}, \quad \theta=\arccos \left(\frac{-q}{2 \rho}\right), \quad \Delta=\left(\lambda+R_{3}(\lambda)\right)^{2}+\frac{4 p(0)}{R_{3}^{\epsilon}(\lambda)}>0 \\
s=-\frac{\lambda}{\epsilon}-\frac{\lambda^{2}}{3}<0, \quad q=\frac{\lambda}{27 \epsilon}\left(2 \lambda^{2} \epsilon-18 \lambda+27 r^{-2}\right) .
\end{array}\right.
$$

Tedious computations then leads to the following expansion :

$$
\begin{gathered}
R_{1}^{\epsilon}(\lambda), R_{3}^{\epsilon}(\lambda)=\mp \frac{\sqrt{\lambda}}{\sqrt{\epsilon}}-\frac{r^{-2}}{2}+O(\sqrt{\epsilon}), \quad R_{2}^{\epsilon}(\lambda)=-\left(\lambda-r^{-2}\right)+O(\epsilon) . \\
K_{1}^{\epsilon}, K_{3}^{\epsilon}=\frac{\lambda^{1 / 4}}{r^{-1} \epsilon^{1 / 4}} \pm \frac{\lambda}{4} \frac{r^{-2}-4 \lambda}{r^{-1} \lambda} \epsilon^{1 / 4}+O\left(\epsilon^{3 / 4}\right), \quad K_{2}^{\epsilon}=-\frac{r^{-1}}{\sqrt{\lambda-r^{-2}}}+O(\sqrt{\epsilon}) .
\end{gathered}
$$


The negative root $R_{2}^{\epsilon}$ is bounded with respect to $\epsilon$ and converges to the single roots which appears in the case $\epsilon=0$ (leading to the eigenvalues $\lambda_{k}^{0}=r^{-2}+(k \pi)^{2}$ ). Precisely, we compute that for all $k \geq 0$

$$
\operatorname{det}\left(M^{\epsilon}\left(\lambda_{k}^{0}\right)\right)=O\left(\epsilon^{1 / 4}\right), \quad \lambda_{k}^{0}=r^{-2}+(k \pi)^{2} \in \sigma\left(\boldsymbol{A}_{\boldsymbol{M}}\right)
$$

so that, from the continuity of the determinant, there exists, for all $k$, an element $\lambda_{k}^{\epsilon,+} \in$ $\sigma\left(\boldsymbol{A}^{\epsilon}\right)$ so that $\lambda_{k}^{\epsilon,+} \rightarrow \lambda_{k}^{0}$ as $\epsilon \rightarrow 0$. But, as suggested by Figure 7 , the spectrum of $\boldsymbol{A}^{\epsilon}$ densifies, as $\epsilon \rightarrow 0$, over the real axis: its appears spurious eigenvalues, say $\left\{\lambda_{k}^{\epsilon,-}\right\}_{k}$ which does not converge toward an element of $\sigma\left(\boldsymbol{A}_{\boldsymbol{M}}\right)$. Consequently, $\sigma\left(\boldsymbol{A}^{\boldsymbol{\epsilon}}\right)$ does not converge punctually toward $\sigma\left(\boldsymbol{A}_{\boldsymbol{M}}\right)$. This is due to the behavior of the roots $R_{1}^{\epsilon}$ and $R_{3}^{\epsilon}$ of order $O(1 / \sqrt{\epsilon})$. Therefore, the functions $\left(\cos \left(r_{1}\left(\lambda^{\epsilon}\right)\right), \sin \left(r_{1}\left(\lambda^{\epsilon}\right)\right)\right)$ where $r_{1}\left(\lambda^{\epsilon}\right)$ behaves like $O\left(1 / \epsilon^{1 / 4}\right)$ are very oscillatory. This phenomenon is the consequence of two properties: the non-empty essential spectrum for the asymptotic operator $\boldsymbol{A}_{\boldsymbol{M}}$ and the degeneracy of the boundary conditions for $\phi_{3}$ (we refer to [23] chapter 10).

The case $\lambda^{\epsilon}<r^{-2}$ leads also to a densification phenomenon. This densification clearly prevents from any uniform spectral gap with respect to $\epsilon$ and explain the loss of controllability, even far away from the value 0 , element of $\sigma_{\text {ess }}\left(\boldsymbol{A}_{\boldsymbol{M}}\right)$, as $\epsilon \rightarrow 0$. As a summary, the uniform controllability of system (1) w.r.t. $\varepsilon$ is expected if and only if the initial data are generated by the eigenfunctions, says $\left\{\boldsymbol{\phi}_{\boldsymbol{k}}^{+, \varepsilon}\right\}_{(k>0)}$, associated with $\lambda_{k}^{\epsilon,+}$, weakly converging in $\boldsymbol{H}$ toward the eigenfunctions of $\boldsymbol{A}_{\boldsymbol{M}}$.
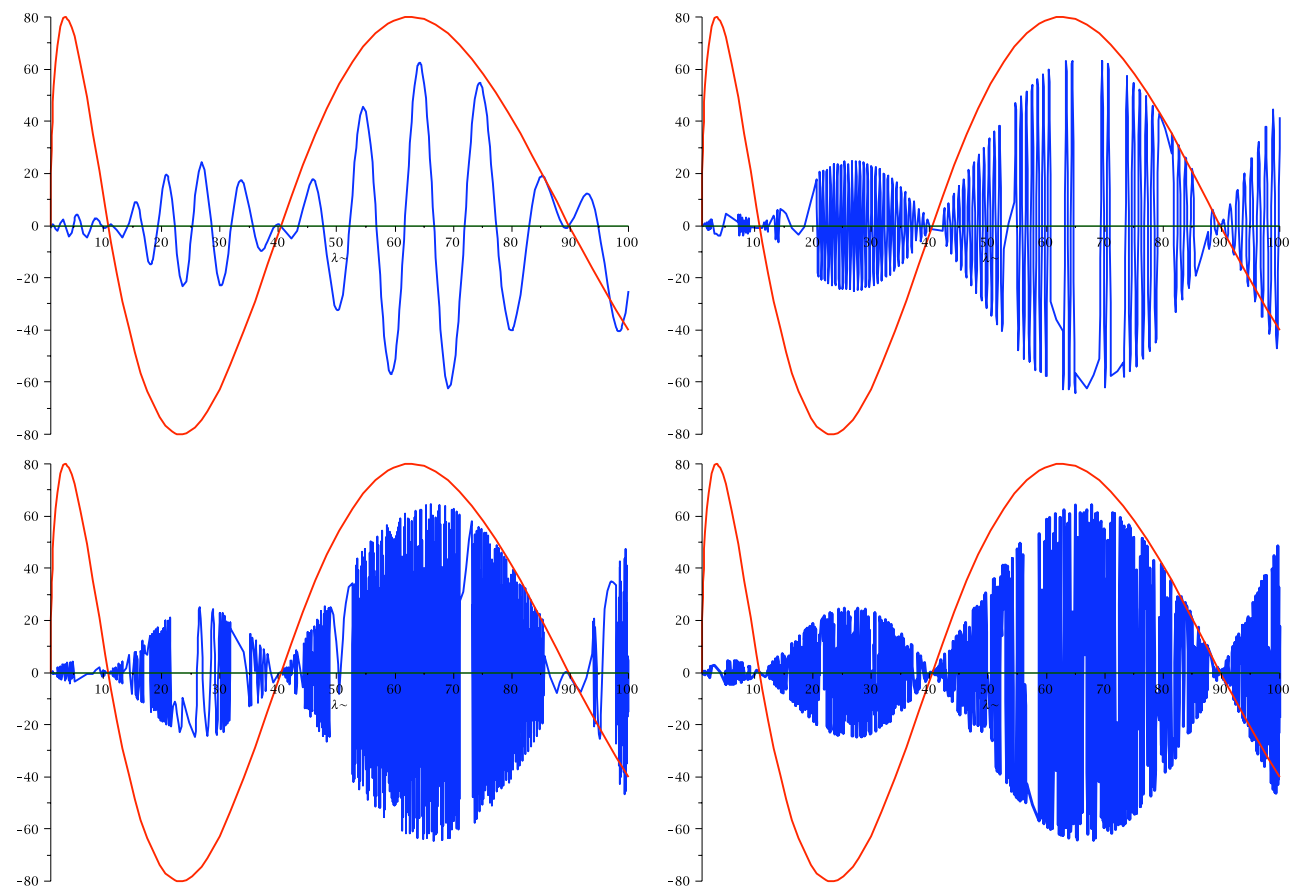

Figure 6: Evolution of $\lambda^{\epsilon} \rightarrow \operatorname{det}\left(M\left(\lambda^{\epsilon}\right)\right)$ and $\lambda^{\epsilon} \rightarrow \sin \left(r_{2}\left(\lambda^{\varepsilon}\right)\right)$ in $\left[r^{-2}, 100\right)$ for $\epsilon=$ $10^{-1}, 10^{-2}, 10^{-3}, 10^{-4}$ and $r^{-1}=1$. 


\section{References}

[1] F. Ammar-Khodja, G. Geymonat and A.Münch, On the exact controllability of a system of mixed order with essential spectrum. C.R.Acad. Sci. Paris série I, 346, 629-634 (2008).

[2] F. Ammar-Khodja, K. Mauffrey and A.Münch, Exact boundary controllability of a system of mixed order with essential spectrum in 2-D, Preprint 2009, Université Blaise Pascal.

[3] C. Castro, S. Micu and A. Münch, Numerical approximation of the boundary control for the wave equation with mixed finite elements in a square. IMA J. Numerical Analysis, 28(1), 186-214 (2008).

[4] S. Chai, Y. Guo and P-F. Yao, Boundary feedback stabilization of shallow shells. SIAM J. Control and Opt., 42(1), 239-259 (2003).

[5] D. Chapelle D and K.J. Bathe, The finite element analysis of shell - Fundamentals. Springer, (2003).

[6] P.G. Ciarlet, Mathematical elasticity. Volume III: Theory of shells. North-Holland, Amsterdam, (2000).

[7] Ph. Destuynder, A classification of thin shell theories. Acta Appl. Math. 4(1) (1985) 15-63.

[8] Ph. Destuynder, Modélisation des coques élastiques minces. Masson, Paris (1990).

[9] G. Geymonat, P. Loreti and V. Valente, Contrôlabilité exacte d'un modèle de coque mince. C.R.Acad.Sci Série I, 313, (1991), 81-86.

[10] G. Geymonat, P. Loreti and V. Valente, Exact controllability of thin elastic hemispherical shell via harmonic analysis. Boundary value problems for partial differential equations and applications, Masson, (1993).

[11] G. Geymonat, P. Loreti and V. Valente, Spectral problems for thin shells and exact controllability. Spectral Analysis of Complex structures, Travaux en cours, 49, Hermann, Paris, 1995, 35-57.

[12] G. Geymonat and V. Valente, A noncontrollability result for systems of mixed order. SIAM J. Control Optim ,39(3), 661-672 (2000).

[13] R. Glowinski, W. Kinton and M.F. Wheeler, A mixed finite element formulation for the boundary controllability of the wave equation. Int. J. Numer. Methods. Eng., 27(3), 623-636 (1989).

[14] I. Lasiecka, R. Triggiani and V. Valente, Uniform stabilization of spherical shells by boundary dissipation. Advances in differential equations, 4, 635-674, (1996).

[15] C. Lebiedzik, Exact boundary controllability of a shallow intrinsic shell model. J. Math. Anal. Appl., 335, 584-614, (2007). 
[16] P. Loreti and V. Valente, Partial exact controllability for spherical membranes. SIAM J. Control Optim, 35(2), 641-653, (1997).

[17] J.E. Lagnese and J.L. Lions, Modelling analysis and control of thin plates. Masson, RMA 6, Paris (1988).

[18] J.L. Lions, Contrôlabilité exacte, stabilisation et perturbations de systèmes distribués, Tome 1. Masson, RMA 8, Paris (1988).

[19] J.L. Lions and E. Magenes, Problèmes aux limites non homogènes et applications, vol. 1. Dunod, Paris (1968).

[20] B. Miara and V. Valente, Exact controllability of a Koiter Shell by a Boundary Action. J. Elasticity 52 267-287 (1999).

[21] A. Münch, A uniformly controllable and implicit scheme for the 1-D wave equation. Mathematical Modelling and Numerical Analysis 39(2), 377-418 (2005).

[22] A. Münch and A. Pazoto, Boundary stabilization on a nonlinear arch : Theoretical vs. Numerical Analysis. Discrete and Continuous Dynamical Systems Series B, 10(1), 197-219 (2008).

[23] J. Sanchez-Hubert and E. Sanchez-Palencia, Coques élastiques minces - Propriétés asymptotiques. RMA Masson, 1997.

[24] V. Valente, Relaxed exact spectral controllability of membrane shells, J. Math. Pures Appl. 76 551-562 (1997).

[25] E. Zuazua, Propagation, Observation, Control and Numerical Approximation of Waves approximated by finite difference methods, SIAM Review, 47(2), 197-243, (2005). 